\title{
Protocolo acelerado de degradação do sistema de reforço EBR-CFRP aplicado em vigas de concreto armado
}

\author{
Accelerated degradation protocol of the EBR-CFRP \\ strengthening system applied to reinforced concrete \\ beams
}

\section{Gláucia Maria Dalfré \\ Luiz Antônio Sarti Júnior \\ Ciro José Ribeiro Villela Araújo}

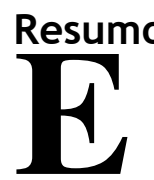

m geral os materiais na indústria da construção civil estão sujeitos à mecanismos de degradação, como alterações químicas, físicas e mecânicas. No âmbito de elementos de concreto armado reforçados com materiais compósitos, verifica-se que a degradação afeta a parte mais sensível do sistema, as resinas à base de epóxi. O presente trabalho é baseado no uso do protocolo de acondicionamento apresentado na norma ACI 440.9R (AMERICAN..., 2015) para avaliação da durabilidade de sistemas de reforço aplicados em vigas de concreto armado reforçadas à flexão com mantas de fibra de carbono, aplicadas segundo a técnica de colagem externa EBR. Os resultados do protocolo de degradação acelerado foram comparados com os obtidos em ensaios de intemperismo natural. Esses demonstraram que os adesivos epoxídicos apresentam grandes reduções em suas propriedades mecânicas, enquanto os compósitos de CFRP permanecem com suas propriedades inalteradas após exposição ao intemperismo ou degradação acelerada. $\mathrm{O}$ sistema de reforço proporciona grandes incrementos de capacidade de carga e rigidez das vigas. Entretanto, verificou-se que os ensaios realizados após um ciclo de degradação acelerado com duração de $1.000 \mathrm{~h}$ (42 dias) e após 6 meses de exposição às intempéries demonstraram redução de aproximadamente $10 \%$ da capacidade de carga dos elementos reforçados, indicando uma possível degradação do sistema de reforço.

Palavras-chave: Vigas de concreto armado. Reforço EBR. CFRP. Degradação acelerada. Intemperismo.

\begin{abstract}
In general, all materials used in the construction industry are subject to the influence of degradation mechanisms, which are characterized by a sequence of chemical, physical, and mechanical changes. In the case of reinforced concrete elements strengthened with composite materials, it is generally observed that degradation affects the most sensitive part of the strengthening system, the epoxy adhesives. This study is based on the use of the Accelerated Conditioning Protocol (ACP) presented by ACI 440.9R (AMERICAN..., 2015) for the evaluation of the durability of reinforced concrete beams flexurally strengthened with CFRP sheets applied according to the EBR technique. The results of the ACP were compared with those obtained through natural weathering tests. The results showed that epoxy adhesives had their mechanical properties significantly reduced, while the CFRP composites remained unchanged after exposure to weathering or to accelerated degradation. The strengthening system greatly increased the load carrying capacity and stiffness of the beams. However, the tests carried out after an accelerated degradation cycle lasting 1,000 h (42 days) and those carried out after 6 months' exposure to weathering showed a reduction of approximately $10 \%$ in the load carrying capacity of the strengthened elements, indicating a possible degradation of the strengthening system.
\end{abstract}

Keywords: Reinforced concrete beams. EBR strengthening. CFRP. Accelerated degradation. Weathering. 


\section{Introdução}

De acordo com Dalfré (2013), Fernandes (2016) e Rezazadeh, Barros e Ramezansefat (2016), estruturas de concreto armado, aço e madeira estão sendo cada vez mais reforçadas por meio de técnicas baseadas no uso de polímeros reforçados com fibras (FRP - fiber reinforced polymer em língua inglesa) em função de uma série de vantagens que incluem suas elevadas propriedades mecânicas, resistência à corrosão, leveza e facilidade de aplicação (KARBHARI, 2007; AMERICAN..., 2017; FÉDÉRATION..., 2019).

A principal técnica de reforço com FRP atualmente utilizada, designada na literatura internacional por EBR (externally bonded reinforcement), consiste na colagem externa de mantas ou laminados do material na superfície do elemento (de concreto, aço ou madeira), permitindo que o reforço seja realizado de forma rápida, com pouca intervenção durante a instalação e sem a necessidade de muitos equipamentos.

Embora as propriedades e vantagens do reforço utilizando FRP descritas anteriormente sejam bem conhecidas, este permanece desprotegido e suscetível às ações ambientais. Em geral, os elementos estruturais reforçados com FRP estão sujeitos a uma variedade de carregamentos e condições de exposição (físicas, químicas ou mecânicas) que podem atuar individualmente ou em sinergia na degradação dos compósitos de FRP.

Os fatores considerados mais críticos para a durabilidade dos sistemas de reforço com FRP são apresentados na Figura 1 (INTELLIGENT..., 2006).

A forma como o FRP irá reagir quando exposto por longos períodos a condições que deterioram suas propriedades físicas e mecânicas dependerá diretamente da composição de seus materiais constituintes (matriz polimérica e fibras utilizadas).

O desempenho e o comportamento no longo prazo dos compósitos dependem da natureza química da matriz, do processo de fabricação, da aplicação e das condições ambientais em serviço. Nesse sentido, o desafio atual para os engenheiros civis está relacionado a questões que envolvem o comportamento desses materiais ao longo do tempo e a durabilidade do sistema de reforço, principalmente quando este fica exposto a condições ambientais agressivas e sem nenhum tipo de proteção.

\section{Estado da arte}

O principal problema relacionado à durabilidade está ligado ao tipo de matriz utilizada na composição dos materiais compósitos de FRP. As resinas à base de epóxi, comumente utilizadas, são afetadas diretamente pelos raios UV, além de possuírem limitações a altas temperaturas e à umidade.

\section{Umidade e temperatura}

O FRP é um material que apresenta características favoráveis a sua utilização em ambientes potencialmente corrosivos por não ser suscetível à corrosão eletromagnética. Entretanto, quando expostos por longos períodos a ambientes de umidade, os FRP podem sofrer degradação em suas propriedades físicas e/ou mecânicas dependendo de sua taxa de absorção de água, a qual dependerá de uma série de fatores, tais como temperatura, taxa de concentração e tipo de líquido no qual está imerso, tipo de fibra utilizada e grau de cura da resina (INTELLIGENT..., 2006).

Figura 1 - Fatores que afetam a durabilidade dos compósitos de FRP

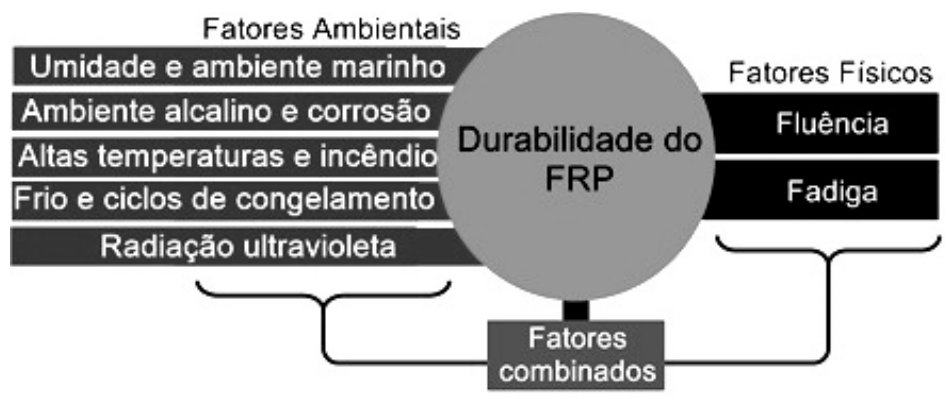

Fonte: ISIS (INTELLIGENT..., 2006).

274 Dalfré, G. M.; Sarti Júnior, L. A.; Araújo, C. J. R. V. 
Segundo Nordin (2003) e Isis (INTELLIGENT..., 2006), a absorção de umidade pelos FRP pode enfraquecer a ligação entre as cadeias poliméricas, causando mudanças reversíveis ou irreversíveis na estrutura dos polímeros, levando a perdas de resistência à tração e redução da rigidez dos compósitos, e promovendo o amolecimento, a plastificação e o inchaço da matriz, o que resulta em perdas de capacidade resistente, módulo de elasticidade e deformação última. A temperatura de transição vítrea $(T g)$ da matriz polimérica também tende a ser reduzida quando os FRP são submetidos à umidade (INTELLIGENT..., 2006). A Figura 2a ilustra um diagrama genérico de ganho de massa em função do tempo para materiais poliméricos expostos à umidade. Tal comportamento também foi verificado nos ensaios realizados por Escobal (2017), tal como apresentado na Figura 2b.

A Figura 3 ilustra um diagrama genérico da evolução da perda de resistência de polímeros submetidos a condições de absorção de umidade em função do tempo.

Yang, Xian e Karbhari (2008) avaliaram o comportamento de corpos de prova de resina epoxídica quando imersos em água deionizada a diferentes temperaturas $\left(23{ }^{\circ} \mathrm{C}, 37,8^{\circ} \mathrm{C}\right.$ e $\left.60^{\circ} \mathrm{C}\right)$, analisados após 24 meses de exposição. A imersão em água deionizada provocou grande alteração da tensão máxima e do módulo de elasticidade, principalmente na avaliação com temperatura de $60{ }^{\circ} \mathrm{C}$. Assim, as reduções médias na tensão máxima de tração e no módulo de elasticidade foram de 43,8\% e de 38,1\% após 24 meses de imersão em água a $23{ }^{\circ} \mathrm{C}$ e $37,8{ }^{\circ} \mathrm{C}$ respectivamente. Para os corpos de prova mantidos a $60{ }^{\circ} \mathrm{C}$, reduções de $69 \%$ e de $68,3 \%$ da tensão máxima de tração e do módulo de elasticidade respectivamente foram obtidas. Tais valores corroboram o apresentado por Frigione (2015), que cita que a penetração excessiva da água é prejudicial aos adesivos epoxídicos porque pode levar à redução da rigidez e da resistência devido à plastificação do material.

Figura 2 - Ganho de massa em polímeros submetidos à umidade

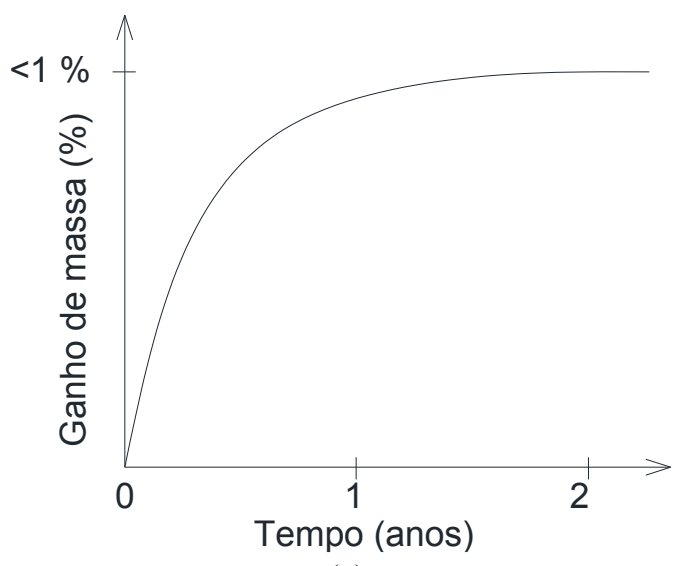

(a)

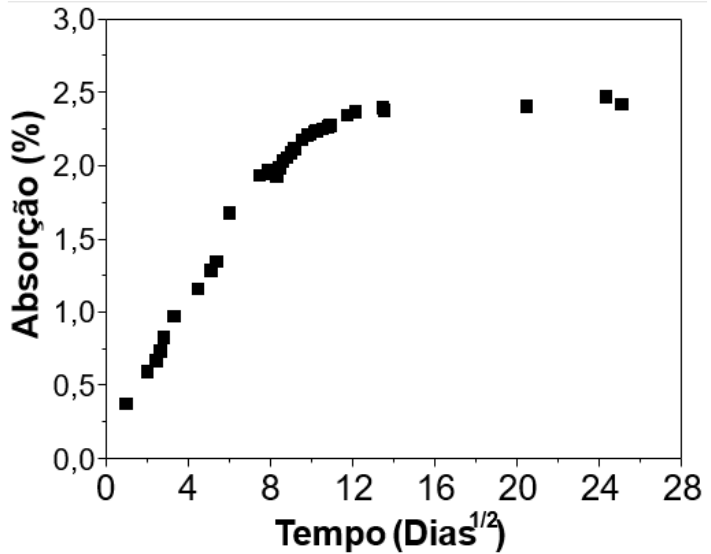

(b)

Fonte: (a) adaptada de ISIS (INTELLIGENT..., 2006) e (b) de Escobal (2017).

Figura 3 - Perda de resistência de polímeros expostos à umidade em função do tempo

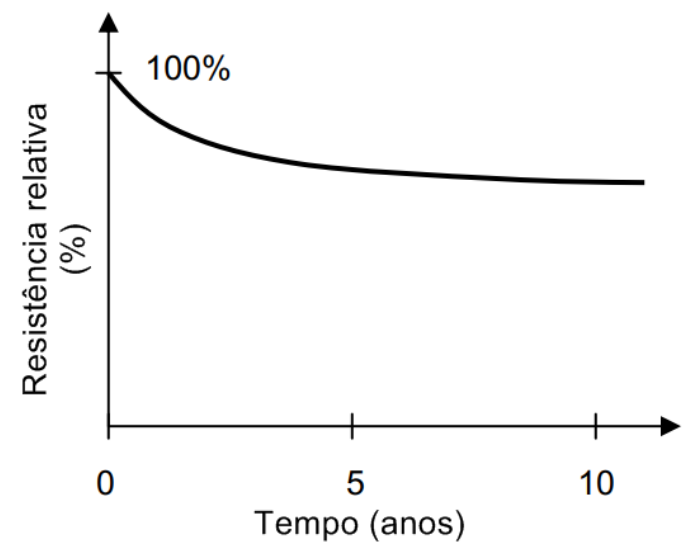

Fonte: ISIS (INTELLIGENT..., 2006). 
Okba et al. (2017) verificaram o comportamento de adesivos epoxídicos diante da elevação de temperatura. Para tal, corpos de prova de resina epoxídica foram imersos a $60{ }^{\circ} \mathrm{C}, 100{ }^{\circ} \mathrm{C}, 150{ }^{\circ} \mathrm{C}$ e $200{ }^{\circ} \mathrm{C}$ pelo período de $1 \mathrm{~h}$ e posteriormente ensaiados. Os resultados obtidos nos ensaios de tração indicaram reduções na resistência à tração de $43 \%, 88 \%, 94 \%$ e $98 \%$ respectivamente após exposição às temperaturas de $60^{\circ} \mathrm{C}, 100$ ${ }^{\circ} \mathrm{C}, 150^{\circ} \mathrm{C}$ e $200^{\circ} \mathrm{C}$.

Escobal (2017) e Dalfré et al. (2019) realizaram ensaios de caracterização de resinas epoxídicas (primer e saturação, denominados de resina A e B respectivamente) nas idades de 7 e 14 dias de cura (referência) e após 4, 8 e 12 meses de imersão contínua ou em ciclos de umidade (7 dias em ambiente seco ou úmido) em água potável. A Figura 4 apresenta o diagrama tensão versus deformação das resinas A e B ao longo do tempo. Os resultados obtidos nos ensaios de tração uniaxial indicam redução da tensão máxima e do módulo de elasticidade de $70 \%$ e $68 \%$ e de $18 \%$ e $38 \%$ para as resinas A e B respectivamente para a idade de 2 anos em imersão contínua. Com relação aos ciclos de umidade, a resina A apresentou reduções da tensão de tração e módulo de elasticidade de até $74 \%$ e $69 \%$ respectivamente, enquanto a resina B apresentou reduções de até $51 \%$ e $43 \%$ da tensão máxima e módulo de elasticidade respectivamente, quando comparado aos resultados obtidos nos ensaios realizados aos 14 dias (referência).

Com relação aos compósitos, pesquisa realizada por Cromwell, Harries e Shahrooz (2010) constatou que laminados de CFRP expostos a ambientes agressivos, tais como umidade constante e solução salina, não apresentaram alterações em sua resistência à tração, módulo de elasticidade e deformação última. Esses resultados também são corroborados em pesquisa realizada por Dalfré (2016), na qual não foi verificada grande alteração na resistência à tração e no módulo de elasticidade de corpos de prova de mantas de CFRP expostos a ciclos de umidade e umidade constante em um período total de 2 anos.

\section{Exposição a raios ultravioleta}

Nos sistemas de reforço com FRP pela técnica EBR os materiais são aderidos aos substratos de concretos dos elementos que se deseja reforçar. A menos que seja realizada uma camada protetora, o FRP fica diretamente exposto ao ambiente em que o elemento estrutural está situado e, consequentemente, a seus possíveis agentes agressivos ou eventualidades. Se esses elementos estruturais estiverem alocados em um ambiente externo, o FRP pode ficar diretamente exposto aos efeitos dos raios ultravioleta (UV), tal como apresentado na Figura 5. Segundo Kabir, Shrestha e Samali (2016), a exposição ao ambiente externo é o principal agente agressivo da ligação entre o concreto, o adesivo e o FRP.

As fibras de carbono e vidro são resistentes às ações dos raios UV. Por outro lado, a matriz polimérica se degrada pelo processo de fotodegradação devido à quebra de ligações químicas entre as cadeias poliméricas pela energia absorvida dos raios UV. Esse processo, isoladamente, age apenas numa pequena camada superficial do FRP, resultando na descoloração, na oxidação da superfície, na fragilização e na microfissuração da matriz polimérica, sem perdas significativas em seu desempenho mecânico. Embora os raios UV degradem o FRP superficialmente, os danos superficiais podem servir como caminho para que outros agentes agressivos penetrem na matriz e nas fibras, podendo levar a reduções significativas nas propriedades mecânicas do FRP (INTELLIGENT..., 2006).

Figura 4 - Diagrama com as curvas médias de tensão versus deformação para as amostras de resinas A (a) e B (b) expostas a umidade constante

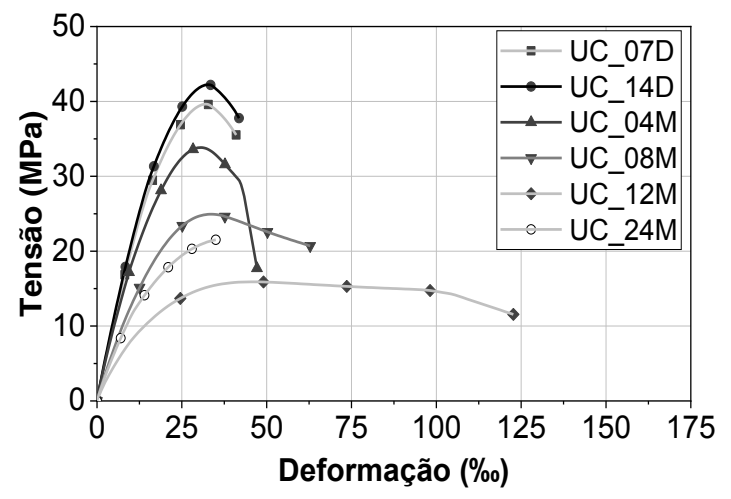

(a)

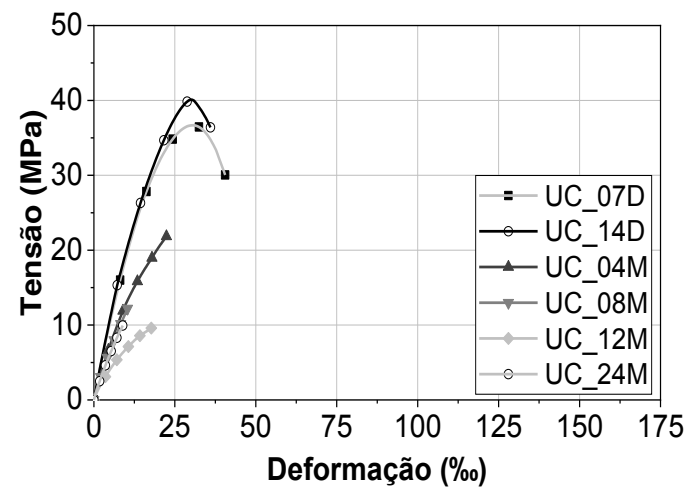

(b)

Fonte: Escobal (2017) e Sarti Júnior (2020).

276 Dalfré, G. M.; Sarti Júnior, L. A.; Araújo, C. J. R. V. 
Figura 5 - Estação de tratamento de esgoto reforçada com CFRP aplicado segundo a técnica EBR

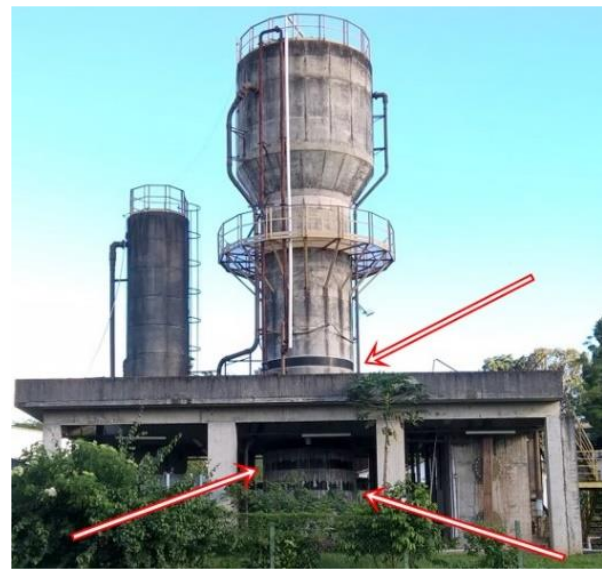

Fonte: Oliveira (2019).

Homam e Sheikh (2000) estudaram o efeito isolado da exposição a raios UV em compósitos de FRP fabricados com resina epoxídica. Corpos de prova de CFRP e de GFRP foram expostos em ambiente de controle, com temperatura de $22{ }^{\circ} \mathrm{C}$ e umidade relativa do ar de $40 \%$, radiação UV por lâmpadas de UV-A em $156 \mathrm{~W} / \mathrm{m}$ e temperatura de $38^{\circ} \mathrm{C}$. Os corpos de prova foram posteriormente ensaiados à tração uniaxial e ao cisalhamento segundo o ensaio single lap bonded. Foram verificados pequenos aumentos de resistência à tração e rigidez nos corpos de prova expostos à radiação UV em relação aos mantidos em ambiente laboratorial para os períodos de exposição de 1.200 h e 4.800 h, para ambos os compósitos. A exposição aos raios UV também não afetou significativamente a resistência ao cisalhamento dos compósitos. Contudo, os pesquisadores relatam grande dispersão nos dados obtidos nesse ensaio.

Zhao et al. (2017) avaliaram corpos de prova de resina epoxídica expostos a ciclos de radiação UV. Para isso, corpos de prova foram expostos à radiação UV $(280 \mathrm{~nm}$ a $315 \mathrm{~nm})$ a uma temperatura entre $57^{\circ} \mathrm{C}$ e 63 ${ }^{\circ} \mathrm{C}$ e umidade entre $90 \%$ e $95 \%$. Os ciclos tiveram duração de 90 dias divididos em 8 h de radiação UV e 4 h de condensação. Ao final dos ensaios, os autores verificaram redução máxima de 20,4\% no módulo de elasticidade dos corpos de prova.

Ferreira (2019) realizou uma análise experimental do comportamento mecânico dos materiais constituintes do sistema de reforço e de vigas de concreto armado reforçadas à flexão com mantas de CFRP aplicadas segundo a técnica EBR, as quais foram mantidas em ambiente laboratorial (interno e protegido) ou expostas a intempéries (exposição exterior). Foram ensaiadas oito vigas de concreto armado com seção retangular de $12 \times 20 \mathrm{~cm}^{2}$, vão livre entre apoios de $230 \mathrm{~cm}$, taxa de armadura longitudinal de $0,75 \%$, e concreto com resistência à compressão de $20 \mathrm{MPa}$. Do conjunto de vigas, quatro foram utilizadas como referência, das quais duas não possuíam reforço e duas foram reforçadas com mantas de fibra de carbono do tipo unidirecional, e as demais foram divididas em dois grupos e expostas por 6 meses em ambiente laboratorial (LAB) e a intempéries (WEA). Segundo o sistema de classificação climática de Köpper-Geiger, o local de realização da pesquisa possui clima local definido como subtropical úmido com inverno seco e verão quente (Cwa). Essas vigas foram submetidas a ensaios de flexão em três pontos com aplicação de força concentrada a meio vão. Para além disso, corpos de provas dos materiais constituintes do sistema de reforço também foram confeccionados, expostos às mesmas condições ambientais das vigas e posteriormente avaliados por meio de ensaios de tração uniaxial. Os resultados demonstraram que os adesivos epoxídicos expostos às intempéries apresentaram reduções de até $70 \%$ em suas propriedades mecânicas, enquanto o compósito de CFRP permaneceu com suas propriedades equivalentes após exposição. Para as vigas de concreto armado reforçadas foi verificado que o sistema de reforço proporciona incrementos de $50 \%$ e $28 \%$ na capacidade de carga e rigidez dos elementos reforçados respectivamente. No entanto, os ensaios realizados após 6 meses de exposição às intempéries demonstraram redução de $10 \%$ no incremento da capacidade de carga dos elementos reforçados. A Figura 6 ilustra os diagramas de força versus deslocamento para os resultados de Ferreira (2019). 
Figura 6 - Comparação entre diagramas força versus deslocamento das vigas sem reforço (0) e reforçadas (CFRP) e expostas aos ambientes (a) laboratorial (LAB) e (b) intempéries (WEA)

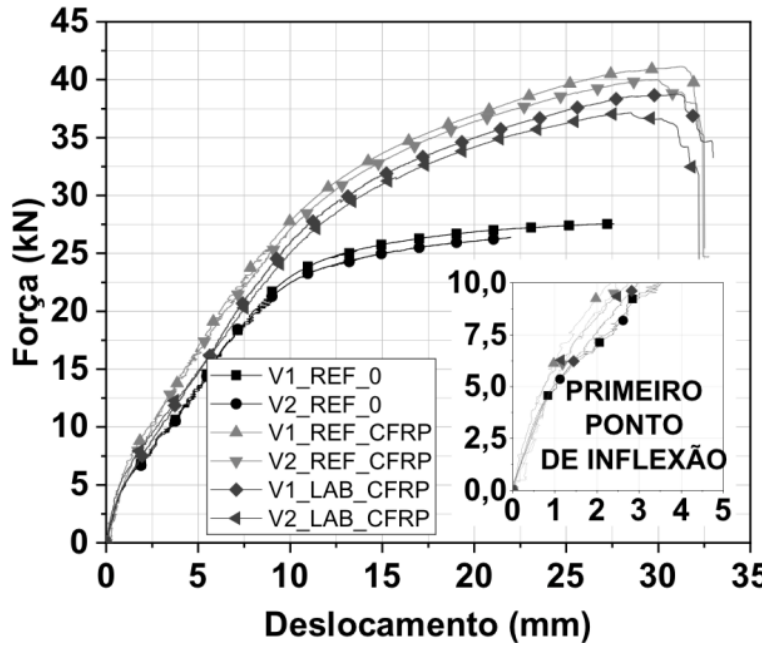

(a)

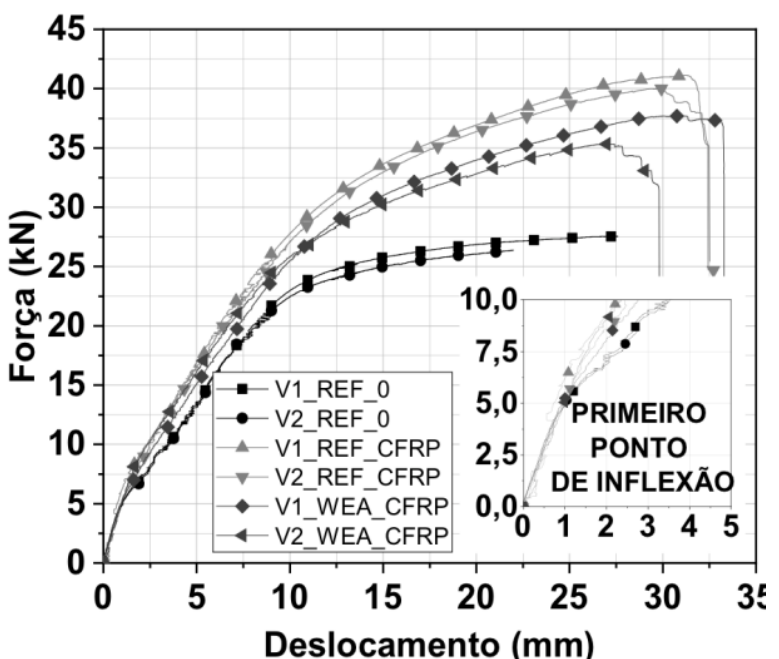

(b)

Fonte: Ferreira (2019).

\section{Protocolo de degradação acelerada}

Geralmente, a taxa de degradação de determinado material em suas condições normais é muito baixa, exigindo que o período de realização do ensaio de verificação do comportamento seja longo o suficiente de modo que seja possível a observação de uma consequente alteração de suas propriedades mecânicas. Nesse sentido, a realização de ensaios acelerados é uma opção, pois geralmente reduzem o período do ensaio, possibilitando a observação, muitas vezes, de níveis consideráveis de degradação.

Os protocolos de ensaios acelerados (ACP, Accelerated Conditioning Protocols em língua inglesa) são baseados na incorporação de variáveis, constantes ou alternadas, tais como temperatura elevada, umidade, voltagem, intemperismo, pressão e reagentes químicos, que tendem a degradar os materiais ou elementos.

Segundo a norma ACI 440.9R (AMERICAN..., 2015), os corpos de prova devem ser mantidos imersos em água potável, com temperatura controlada de $50{ }^{\circ} \mathrm{C} \pm 3{ }^{\circ} \mathrm{C}$, e posteriormente removidos e ensaiados após períodos de exposição que variam entre $1.000 \mathrm{~h}$ e $10.000 \mathrm{~h}$. A Figura 7 apresenta o esquema de ensaio proposto para a realização do protocolo de acondicionamento.

Tal norma recomenda ainda a avaliação das propriedades após um período de exposição intermediário de 3.000 h. Com relação aos ensaios de caracterização das propriedades dos materiais e elementos, estes devem ser conduzidos dentro do intervalo de $2 \mathrm{~h}$ a $5 \mathrm{~h}$ após a remoção dos corpos de prova dos ambientes de degradação.

\section{Programa experimental}

O comportamento de vigas de concreto armado reforçadas com FRP é influenciado por uma série de fatores tais como a geometria do elemento reforçado, a resistência à compressão do concreto, a taxa de armadura, a taxa e o tipo de fibras utilizadas no compósito de FRP, a matriz polimérica utilizada, a configuração do sistema de reforço, a técnica de reforço utilizada e o ambiente ao qual a estrutura está exposta. Nesse sentido, este trabalho avalia o comportamento de materiais e de vigas de concreto armado, reforçadas com o uso da técnica EBR-CFRP, quando expostas a degradação, seguindo o protocolo de ensaio acelerado baseado na recomendação ACI 440.9R (AMERICAN..., 2015).

Para isso, as vigas e os materiais intervenientes (resinas epoxídicas e compósitos de CFRP) foram acondicionados em dois cenários distintos:

(a) ambiente laboratorial (interno, protegido); e

(b) tanques de acondicionamento para avaliação da durabilidade (com imersão do sistema de reforço em água potável, obtida da rede pública de abastecimento, com temperatura constante de $50{ }^{\circ} \mathrm{C} \pm 3{ }^{\circ} \mathrm{C}$. 
Figura 7 - Arranjo de ensaio proposto pelo código americano $\mathrm{ACl}$ para exposição de corpos de prova à degradação acelerada

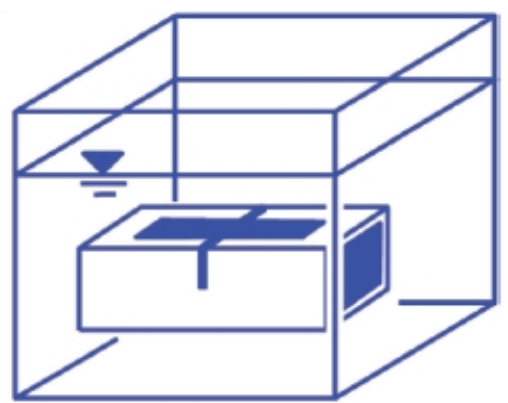

Fonte: adaptada de ACI 440.9R (AMERICAN..., 2015).

As principais fases da campanha experimental serão apresentadas a seguir.

\section{Geometria das vigas, ambientes de degradação, sistema de reforço e configuração do ensaio de flexão}

\section{Vigas}

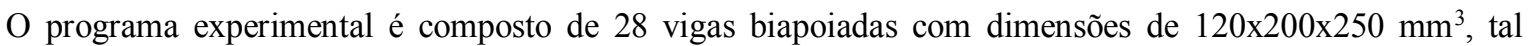
como apresentado na Figura 8 , as quais possuem concreto com resistência à compressão ( $\mathrm{f}_{\text {ck }}$ ) de $32 \mathrm{MPa}$, armadura de flexão positiva composta de duas barras de aço CA-50 com diâmetro de $10 \mathrm{~mm}$ (taxa de armadura longitudinal de $0,75 \%$ ) e porta-estribos composto de duas barras com diâmetro de 6,3 mm. Para evitar a ruptura por cisalhamento, estribos de aço CA-60 com diâmetro de $5 \mathrm{~mm}$ e espaçamento de $10 \mathrm{~cm}$ foram utilizados. As vigas foram dimensionadas no domínio 2 de deformações, tal como o apresentado na norma NBR 6118 (ABNT, 2014), com o uso do diagrama simplificado de distribuição de tensões e deformações de uma viga com seção retangular e armadura simples (FERREIRA, 2019).

\section{Sistema de reforço EBR-CFRP}

Entre as 28 vigas, 14 não possuíam nenhum tipo de reforço e as demais foram reforçadas à flexão com uma camada de manta de CFRP aplicada segundo a técnica EBR. Nesse sentido, uma camada de manta unidirecional de fibras de carbono com gramatura de $300 \mathrm{~g} / \mathrm{m}$ e espessura de $0,176 \mathrm{~mm}$ foi cortada em faixas com largura de $11 \mathrm{~cm}$ e comprimento de $220 \mathrm{~cm}$, de modo que suas dimensões se adequassem à superfície tracionada da viga de concreto armado (Figura 9).

As faixas de manta de CFRP foram aplicadas externamente às vigas após a cura do concreto, seguindo a metodologia típica de reforço. Na execução do sistema de reforço EBR-CFRP, a superfície de concreto foi primeiramente preparada de modo a garantir a perfeita aderência entre a manta de CFRP e o substrato de concreto. O substrato foi desbastado com um disco de desbaste diamantado acoplado a uma esmerilhadeira. Foi removida toda a camada de nata de cimento até a exposição dos agregados. Em seguida se aplicou ar comprimido sobre a superfície para limpeza e eliminação das partículas sólidas. Após a preparação da superfície, aplicou-se uma camada da resina primer de forma a melhorar as condições de aderência entre o CFRP e o substrato de concreto. Procedeu-se à colagem do CFRP com o auxílio da resina epoxídica de saturação, a qual foi aplicada com intervalo de 40 min após a aplicação do primer. O preparo das resinas do tipo primer e de saturação foi baseado em três passos: agitação de cada um dos componentes; mistura do componente A ao B na proporção indicada pelo fabricante; e mistura mecânica até a obtenção de uma coloração uniforme. A resina primer foi espalhada na superfície de concreto com o auxílio de um pincel. Fez-se também a impregnação da manta de CFRP com a resina de saturação e, antes da aplicação do sistema de reforço, fez-se a aplicação dessa resina ao substrato de concreto. Na aplicação do reforço procurou-se garantir o alinhamento das fibras na direção longitudinal da viga, a não formação de bolhas de ar no tardoz do compósito de CFRP e o não acúmulo de resina em excesso. Os passos e procedimento utilizados no reforço das vigas de concreto armado são apresentados na Figura 10. 
Figura 8 - Característica das vigas de concreto armado (unidades em $\mathrm{cm}$ )

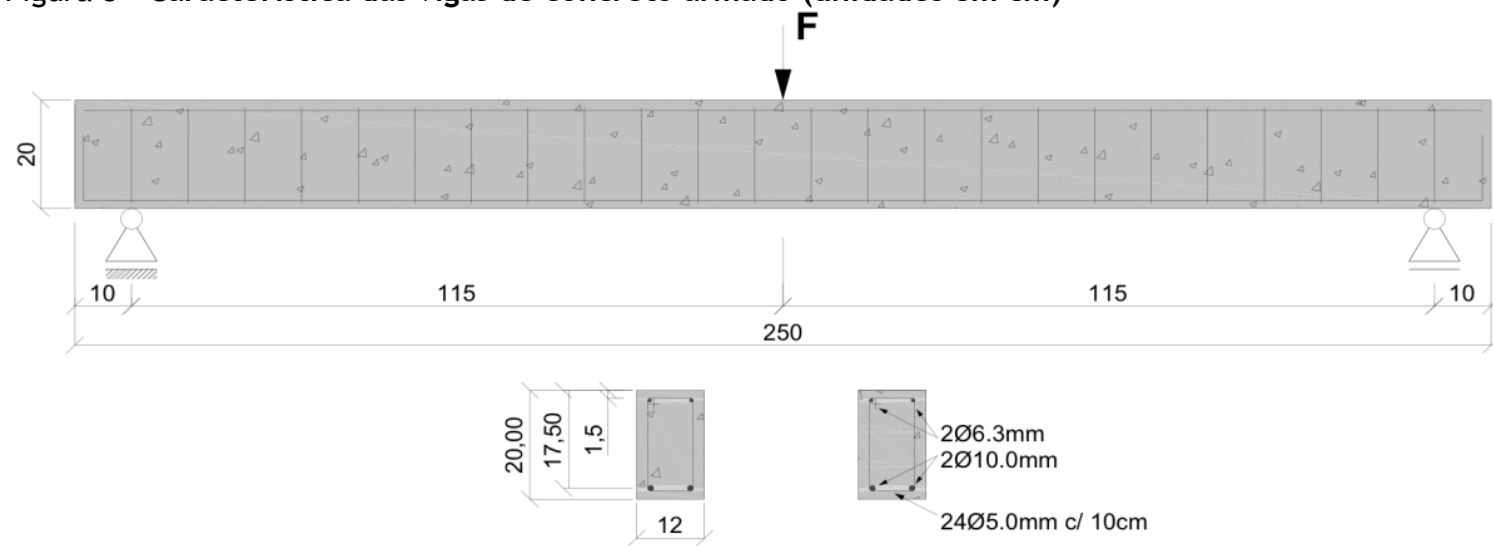

Fonte: Ferreira (2019) e Sarti Júnior (2020).

Figura 9 - Detalhes do sistema de reforço (unidades em $\mathrm{cm}$ )

$F$

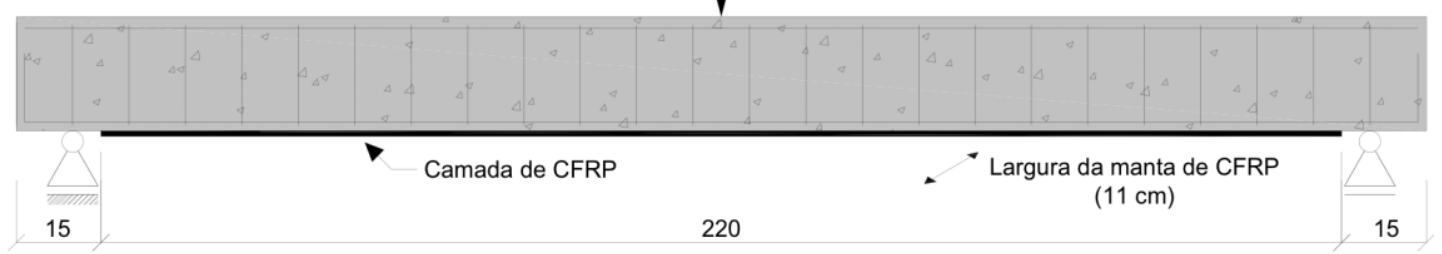

Fonte: Ferreira (2019) e Sarti Júnior (2020).

Figura 10 - Procedimento de aplicação do sistema de reforço nas vigas de concreto armado

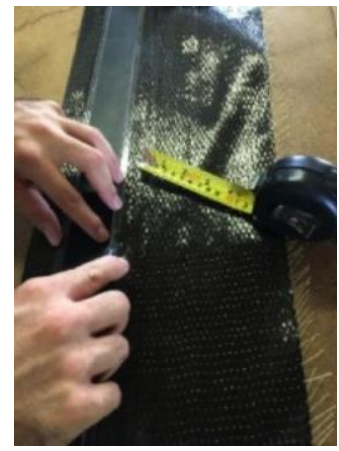

(a) Corte da manta de fibra de carbono

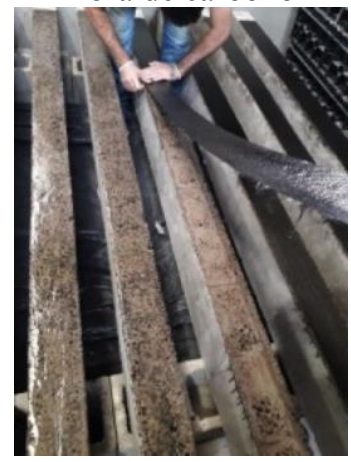

(e) Aplicação do CFRP ao substrato de concreto Fonte: Sarti Júnior (2020).

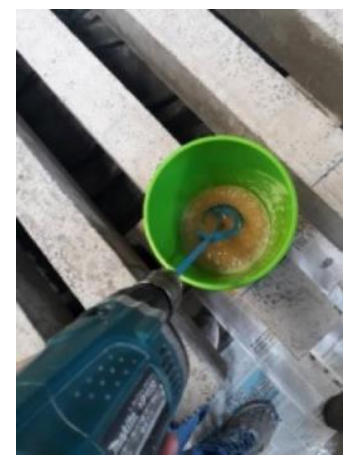

(b) Preparo do primer

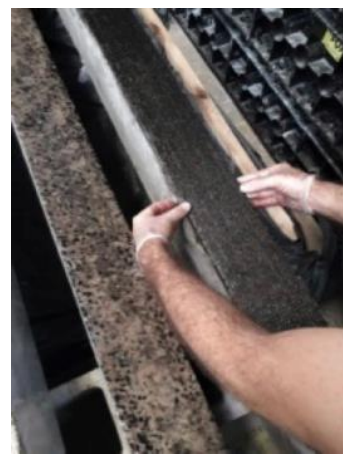

(f) Aplicação do CFRP ao substrato de concreto

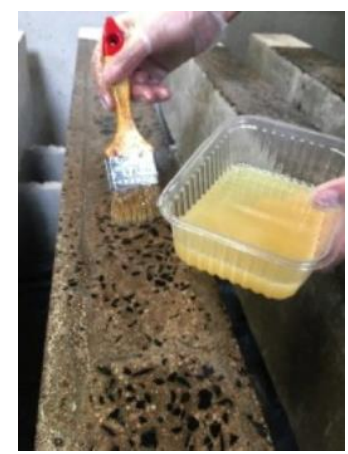

(c) Aplicação do primer

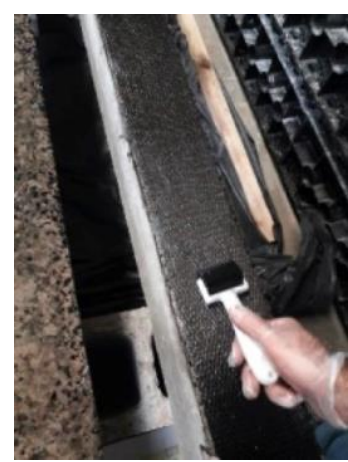

(g) Remoção de bolhas e excesso de resina

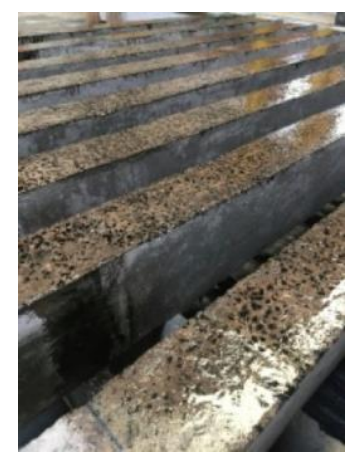

(d) Aplicação do primer

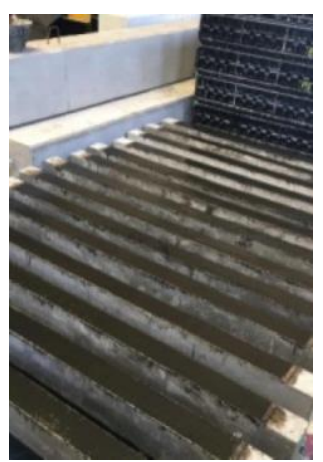

(h) Aspecto final dos elementos reforçados

280 Dalfré, G. M.; Sarti Júnior, L. A.; Araújo, C. J. R. V. 


\section{Caracterização dos materiais}

A caracterização dos materiais foi realizada com o intuito de avaliar as propriedades mecânicas dos materiais utilizados neste programa experimental, especificamente concreto, aço, compósito de CFRP e resinas epoxídicas.

As propriedades mecânicas do aço foram avaliadas por meio de ensaios de tração axial de acordo com as recomendações da norma NBR 6892-1 (ABNT, 2018a). Um mínimo de três amostras para cada diâmetro de barra, as quais possuíam comprimento de $50 \mathrm{~cm}$ e foram aleatoriamente escolhidas, foi ensaiado. Tanto os ensaios de caracterização do aço quanto do concreto foram realizados no Laboratório de Sistemas Estruturais (LSE) da Universidade Federal de São Carlos (UFSCar).

A caracterização do concreto incluiu a análise da resistência à compressão axial e do módulo de elasticidade. Os procedimentos de moldagem e cura foram realizados conforme prescrito pela norma NBR 5738 (ABNT, 2015), sendo moldados corpos de prova cilíndricos com $200 \mathrm{~mm}$ de altura e $100 \mathrm{~mm}$ de diâmetro. Após $24 \mathrm{~h}$ da concretagem, os corpos de prova foram desmoldados e posicionados no mesmo ambiente das vigas. Posteriormente, fez-se a caracterização do material segundo as recomendações das normas NBR 5739 (ABNT, 2018b) e NBR 8522 (ABNT, 2018c).

O sistema de fabricação do compósito de CFRP, no qual a fibra e a matriz são fornecidas em separado, é conhecido como sistema curado in situ, o qual foi utilizado neste programa experimental. Assim, uma manta de fibra de carbono unidirecional com gramatura de $300 \mathrm{~g} / \mathrm{m}$ foi impregnada com resina do tipo epóxi. Ensaios de tração uniaxial na direção principal das fibras foram realizados segundo a ISO 527-1 (INTERNATIONAL..., 2012a) e a ISO 527-5 (INTERNATIONAL..., 2009) para a determinação do módulo de elasticidade e tensão última respectivamente.

Os adesivos utilizados para fixar o sistema de reforço ao substrato de concreto foram fornecidos pelo mesmo fabricante da manta de fibra de carbono utilizada neste trabalho. Neste programa experimental utilizaram-se resinas epoxídicas bicomponentes do tipo primer e saturação. A geometria, dimensões dos corpos de prova e ensaio seguiram as recomendações da norma ISO 527-2 (INTERNATIONAL..., 2012b). Os ensaios de caracterização das resinas epoxídicas e dos compósitos de CFRP foram realizados no Laboratório de Polímeros do Departamento de Engenharia de Materiais (DEMa) da UFSCar. Mais informações sobre a caracterização dos adesivos e resultados experimentais podem ser encontradas no trabalho de Sarti Júnior (2020).

\section{Ambientes de degradação}

Para avaliar o comportamento e durabilidade em longo prazo das vigas e dos materiais constituintes do sistema de reforço, dois ambientes de exposição foram adotados nesta pesquisa (Figura 11):

(a) ambiente laboratorial (LAB): interno e protegido, o qual serviu como referência aos demais ensaios; e

(b) tanques de acondicionamento para avaliação da durabilidade (ACP): com imersão do sistema de reforço em água potável, obtida da rede pública de abastecimento, com temperatura constante de $50^{\circ} \mathrm{C} \pm 3{ }^{\circ} \mathrm{C}$.

$\mathrm{O}$ estudo em andamento é composto de períodos de exposição de $1.000 \mathrm{~h}, 3.000 \mathrm{~h}$ e $10.000 \mathrm{~h}$. Entretanto, este trabalho somente apresenta e discute os resultados obtidos para o período de $1.000 \mathrm{~h}$ - os demais resultados serão apresentados em trabalhos futuros. Para além disso, as recomendações do código americano ACI 440.9R (AMERICAN..., 2015) foram adaptadas para elementos de maiores dimensões, neste caso, vigas de concreto armado.

Tendo em vista tais ambientes, a designação utilizada para identificação de cada viga foi Vx_y_w-z, onde " $\mathrm{x}$ " é o número do elemento ensaiado (1 ou 2), "y" corresponde aos elementos mantidos em ambiente laboratorial (LAB, de laboratory) ou expostos ao protocolo de degradação acelerado (ACP, de Acelerated Conditioning Protocol), "w" indica a utilização ou não de material de reforço (0 ou CFRP) e " $\mathrm{z}$ " corresponde à data de realização do ensaio (14 dias ou $1.000 \mathrm{~h}$ (87 dias) após a aplicação do sistema de reforço). Um resumo do programa experimental é apresentado na Tabela 1.

Paralelamente à campanha experimental das vigas de concreto armado reforçadas com mantas de CFRP foram analisados os materiais constituintes do sistema de reforço, especificamente resinas epoxídicas e compósito de CFRP, nas mesmas idades das vigas. As vigas foram posicionadas no tanque de degradação quando o sistema de reforço atingiu a idade de 14 dias. 
Figura 11 - Ambientes de exposição de vigas e corpos de prova

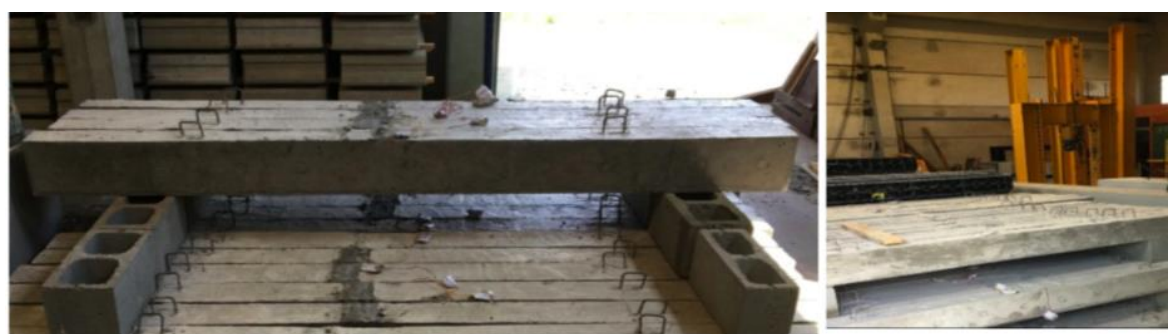

(a) Vigas mantidas em ambiente laboratorial

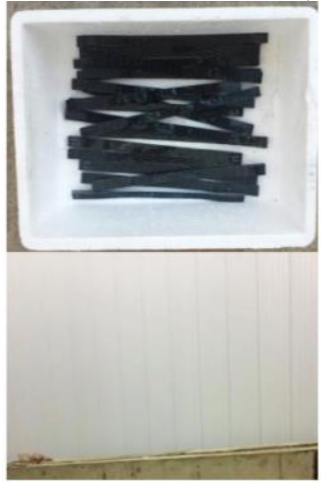

(b) Corpos de prova em ambiente laboratorial

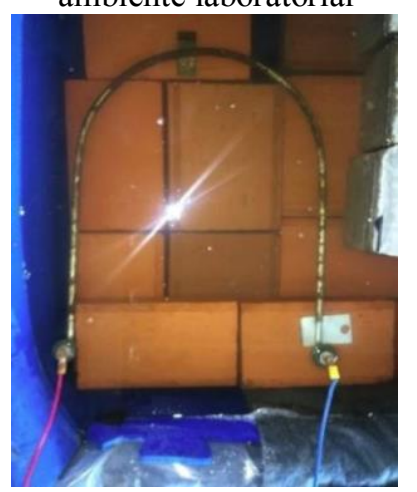

(d) Resistência elétrica utilizada para o aquecimento da água

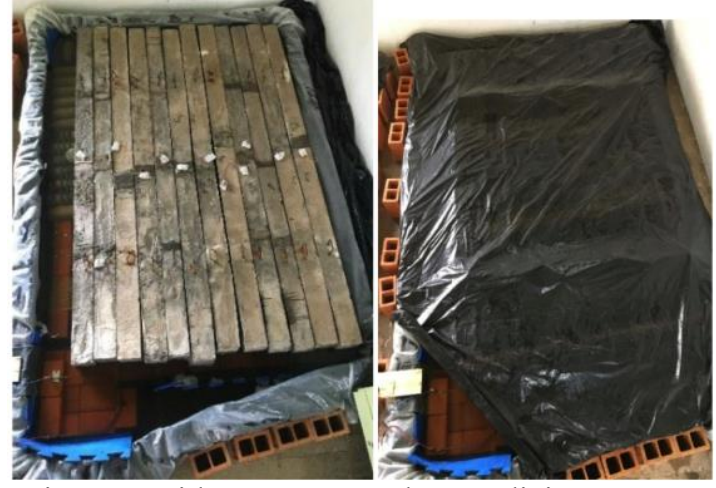

(c) Vigas mantidas em tanque de acondicionamento para avaliação de durabilidade

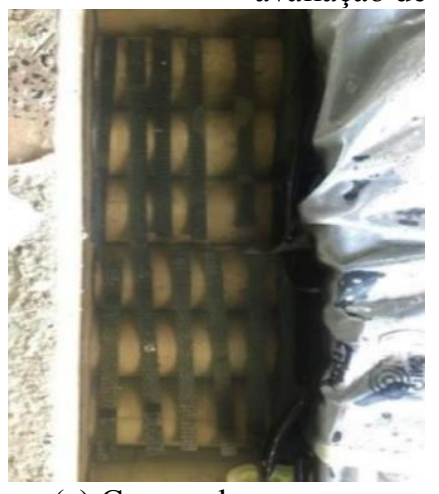

(e) Corpos de prova em tanque de acondicionamento

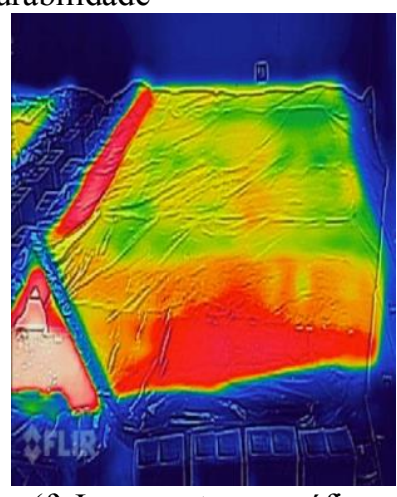

(f) Imagem termográfica para avaliação de temperatura do tanque

Fonte: Sarti Júnior (2020).

Tabela 1 - Resumo do programa experimental

\begin{tabular}{|c|c|c|c|}
\hline Idade do ensaio & Ambiente de exposição & Identificação & Total \\
\hline \multirow{2}{*}{$\begin{array}{c}45 \text { dias (ou } 14 \text { dias após } \\
\text { aplicação do sistema de reforço) }\end{array}$} & \multirow{2}{*}{$\begin{array}{l}\text { Ambiente laboratorial } \\
\text { (Referência) }\end{array}$} & \multirow{2}{*}{$\begin{array}{c}\text { V1_LAB_0_14d } \\
\text { V2_LAB_0_14d } \\
\text { V1_LAB_CFRP_14d } \\
\text { V2_LAB_CFRP_14d }\end{array}$} & 2 \\
\hline & & & 2 \\
\hline \multirow{4}{*}{$\begin{array}{c}87 \text { dias (1.000 h ou } 42 \text { dias de } \\
\text { exposição) }\end{array}$} & \multirow{2}{*}{ Ambiente laboratorial } & \multirow{2}{*}{$\begin{array}{l}\text { V1_LAB_0_1000h } \\
\text { V2_LAB_0_1000h } \\
\text { V1_LAB_CFRP_1000h } \\
\text { V2_LAB_CFRP } 1000 \mathrm{~h}\end{array}$} & 2 \\
\hline & & & 2 \\
\hline & \multirow{2}{*}{$\begin{array}{l}\text { Tanques de acondicionamento } \\
\text { com temperatura controlada }\end{array}$} & \multirow{2}{*}{$\begin{array}{c}\text { V1_ACP_0_1000h } \\
\text { V2_ACP_0_1000h } \\
\text { V1_ACP_CFRP_1000h } \\
\text { V2_ACP_CFRP_1000h }\end{array}$} & 2 \\
\hline & & & 2 \\
\hline
\end{tabular}

Fonte: Sarti Júnior (2020). 


\section{Ensaios das vigas de concreto}

Foram realizados ensaios de flexão em três pontos com aplicação de carga a meio vão das vigas nas idades de (i) 14 dias após a aplicação do sistema de reforço e (ii) após o período de exposição de $1.000 \mathrm{~h}$ (42 dias) dos elementos ao protocolo de acondicionamento baseado nas recomendações da norma ACI 440.9R (AMERICAN..., 2015). Tanto as vigas quanto os corpos de prova de resina ou compósitos foram ensaiados $5 \mathrm{~h}$ após sua retirada dos tanques, tal como preconizado pelo código americano.

Os ensaios das vigas foram conduzidos com o uso de uma máquina universal de ensaios EMIC, modelo DL 60000, disponível no LSE, baseados na metodologia utilizada por Ferreira (2019).

O carregamento foi aplicado sob controle de deslocamento a uma taxa de $1,0 \mathrm{~mm} / \mathrm{min}$. A aplicação de carga foi duplamente aferida com o uso de uma célula de carga externa com capacidade de $200 \mathrm{kN}$ e resolução de leitura de $0,01 \mathrm{kN}$.

Os deslocamentos e as deformações no concreto, armadura longitudinal e compósitos de CFRP foram registradas por meio de um sistema de aquisição de dados modelo ADS-2000, fabricado pela Lynx. A instrumentação das vigas incluiu um transdutor de deslocamento e seis extensômetros elétricos.

O transdutor de deslocamento, da marca Vishay, com campo de leitura de $100 \mathrm{~mm}$, foi utilizado para medir o deslocamento vertical das vigas. Esse foi fixado a um suporte externo e posicionado a meio vão nas vigas.

As deformações no concreto foram medidas por meio de um extensômetro elétrico do tipo BX-120-50AA (11) (resistência de $120 \Omega$ e comprimento da grade de leitura de $50 \mathrm{~mm}$ ), produzido pela Excel Sensores, o qual foi posicionado a meio vão das vigas (SG1).

As deformações na armadura longitudinal foram aferidas por meio de extensômetros elétricos do tipo KFG10-120-C11 (resistência de $120 \Omega$ e comprimento da grade de leitura de $10 \mathrm{~mm}$ ) produzidos pela Kyowa, os quais foram posicionados na seção da seção central da uma das armaduras longitudinais (SG2).

Por fim, as deformações dos compósitos de CFRP foram registradas com o uso de extensômetros elétricos do tipo KFG-05-120-C11 (resistência de $120 \Omega$ e comprimento da grade de leitura de $5 \mathrm{~mm}$ ), também produzidos pela Excel Sensores, os quais foram posicionados ao longo do material de reforço (SG3 ao SG5). As Figuras 12 e 13 apresentam a configuração da instrumentação utilizada no programa experimental.

\section{Resultados e discussões}

Nesta seção são apresentados os resultados dos ensaios de caracterização dos materiais e do comportamento das vigas de concreto sem reforço e daquelas reforçadas com uma camada de manta e CFRP.

\section{Propriedades mecânicas dos materiais}

\section{Concreto}

Os ensaios de caracterização do concreto utilizado neste programa experimental foram realizados 28 dias após a concretagem e, também, após a data da realização dos ensaios das vigas mantidas em ambiente laboratorial ou expostas ao protocolo acelerado, os quais foram realizados nas idades de 45 e 87 dias (após $1.000 \mathrm{~h}$ de imersão no tanque de degradação). Os resultados demonstraram valores médios de resistência à compressão de $32,7 \mathrm{MPa}( \pm 6,9 \%)$ e de $32,7 \mathrm{MPa}( \pm 10,2 \%)$, obtidos aos 28 e 45 dias respectivamente. Com relação aos ambientes de exposição, valores médios de resistências à compressão de $33,4 \mathrm{MPa}( \pm 2,8 \%)$ e de $35,7 \mathrm{MPa}( \pm 2,7)$ e módulos de elasticidade de $34,7 \mathrm{GPa} \quad( \pm 1,1 \%)$ e de $38,2 \mathrm{GPa}( \pm 1,1 \%)$ foram obtidos para o ambiente laboratorial e tanque de acondicionamento respectivamente, para a idade de 87 dias. Os valores entre parênteses representam o coeficiente de variação $(\mathrm{COV})$ das amostras, onde COV $=$ (desviopadrão/média) x 100. Pela análise dos resultados obtidos, percebe-se que valores similares de resistência à compressão e módulo de elasticidade foram obtidos, não tendo indicado degradação do material ante o meio de exposição.

\section{Aço}

Para o aço com diâmetro de $10 \mathrm{~mm}$, verificou-se comportamento típico do aço do tipo CA-50, com patamar de escoamento bem definido (Figura 14), apresentando tensão média de escoamento de 565,0 MPa $( \pm 1,1 \%$ ), deformação média no escoamento de 3,0 \%o ( $(1,8 \%)$, tensão máxima de $578,5 \mathrm{MPa}( \pm 2,8 \%)$ e módulo de elasticidade de 196,4 ( $\pm 1,0 \%)$ GPa. 
Figura 12 - Instrumentação utilizada nas vigas de concreto armado com e sem reforço (unidades em cm)

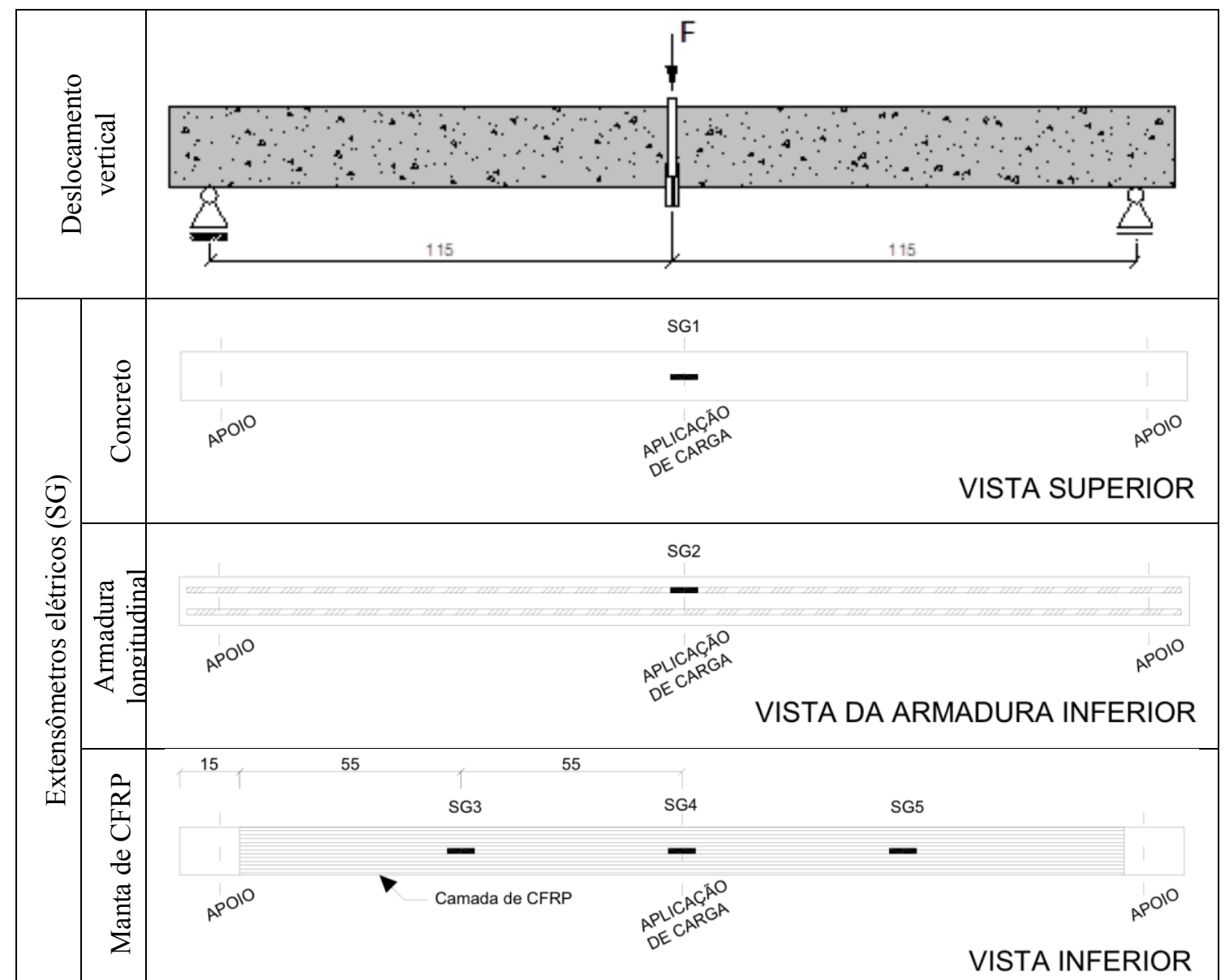

Fonte: Ferreira (2019) e Sarti Júnior (2020).

Figura 13 - Instrumentação posicionada na viga de concreto armado
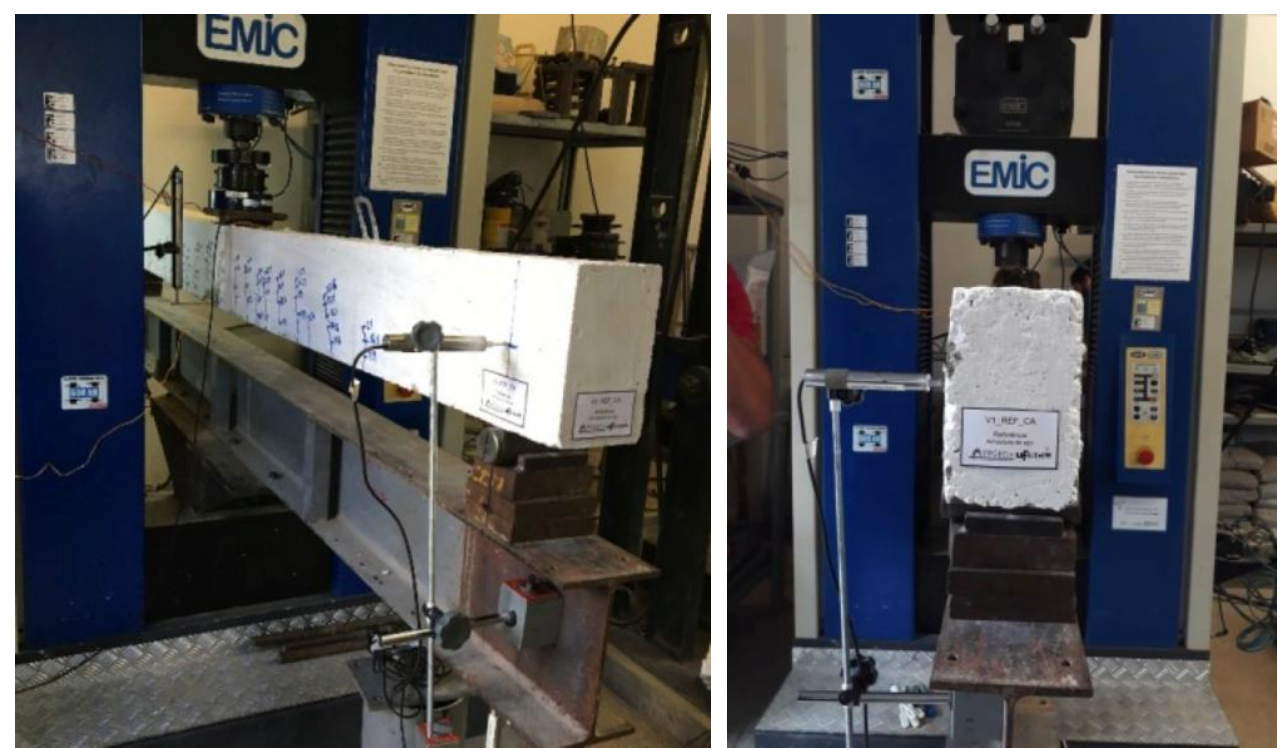

Fonte: Sarti Júnior (2020). 
Figura 14 - Tensão versus deformação do aço da armadura longitudinal de tração

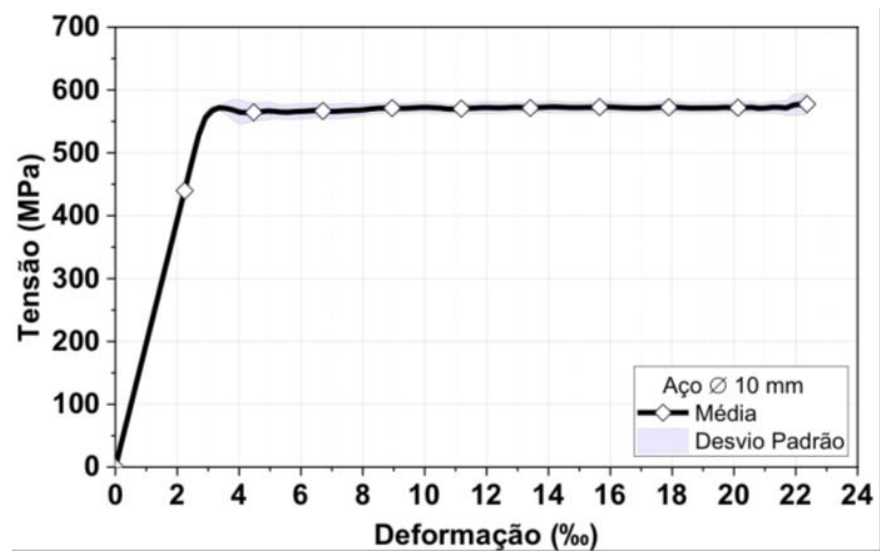

Fonte: Sarti Júnior (2020).

\section{Resinas epoxídicas e compósitos de CFRP}

O comportamento das resinas epoxídicas do tipo primer e de saturação, aqui denominadas de A e B respectivamente, e dos compósitos de CFRP, acondicionados em ambiente laboratorial ou expostos ao protocolo de degradação acelerada, também foi avaliado por meio de ensaios de tração uniaxial realizados nas idades de 14 dias e 42 dias $(1.000 \mathrm{~h})$.

A resina A (primer) mantida em laboratório apresentou módulos de elasticidade e tensão máximas similares para as idades de 14 dias e 42 dias. Entretanto, as resinas expostas ao protocolo de degradação acelerado e ensaiadas aos 42 dias apresentaram reduções de 63\% da força máxima e de $61 \%$ do módulo de elasticidade. Com relação à resina $\mathrm{B}$ (saturação), tendo como referência a idade de 14 dias, reduções de $22,6 \%$ e de $47,6 \%$ na tensão máxima e no módulo de elasticidade respectivamente foram observadas para as resinas mantidas em ambiente laboratorial e ensaiadas com idade de 42 dias. Quando exposta ao protocolo acelerado, e tendo como referência o ambiente laboratorial e idade de 42 dias, uma redução de $50 \%$ da tensão máxima foi obtida, sem alteração do módulo de elasticidade.

Tais resultados corroboram os obtidos por Yang, Xian e Karbhari (2008), Frigione (2015) e Escobal (2017), os quais indicam redução da resistência e rigidez devido à incidência de umidade e temperatura sobre a matriz polimérica.

Com relação aos compósitos, verifica-se que os corpos de prova apresentaram um comportamento elástico linear até a ruptura, típico de materiais frágeis. Pela análise dos resultados, também se verificam reduções insignificativas (cerca de 6\%) da tensão máxima e do módulo de elasticidade na comparação entre os ensaios acelerados e o ambiente laboratorial. Mesmo comportamento foi reportado por Cromwell, Harries e Shahrooz (2010), Dalfré (2016) e Okba et al. (2017).

\section{Comportamento das vigas de concreto}

O comportamento das vigas de concreto armado de referência (com e sem reforço), ensaiadas 14 dias após a aplicação do sistema de reforço (45 dias) ou após 1.000 h (87 dias) de exposição de vigas ao ambiente laboratorial ou ao protocolo de acondicionamento apresentado pela norma ACI 440.9R (AMERICAN..., 2015) foi analisado com base em ductilidade, incremento da capacidade de carga, deformação dos materiais (concreto, aço e CFRP) e modo de ruptura do sistema de reforço.

O critério de parada adotado nos ensaios nas vigas de referência sem reforço foi estabelecido em termos de deslocamento vertical das vigas (no momento em que o valor de $\pm 35 \mathrm{~mm}$ foi atingido), enquanto o adotado nas vigas reforçadas foi baseado na falha do sistema de reforço, seguido pela perda repentina de força e destacamento do material.

A Tabela 2 apresenta o resumo dos resultados médios obtidos nos ensaios das vigas para o início do escoamento da armadura longitudinal $\left(\varepsilon_{s y}\right)$, indicando a força $\left(F_{s y}\right)$, o deslocamento vertical $\left(u_{s y}\right)$ e as deformações no concreto $\left(\varepsilon_{c, s y}\right)$, e no CFRP $\left(\varepsilon_{f, s y}\right)$ para esse momento. Para o início do esmagamento do concreto $\left(\varepsilon_{c, e s m}\right)$ também são apresentados força $\left(F_{c, e s m}\right)$, deslocamento vertical $\left(u_{e s m}\right)$ e deformações no 
aço $\left(\varepsilon_{s, e s m}\right)$, e no CFRP $\left(\varepsilon_{f, e s m}\right)$ respectivamente. Por fim, para a força máxima $\left(F_{\text {max }}\right)$, indica-se o deslocamento vertical registrado nesse momento $\left(u_{\text {,Fmax }}\right)$, assim como as deformações do aço $\left(\varepsilon_{s, F \max }\right)$, do concreto $\left(\varepsilon_{c, F \max }\right)$ e do CFRP $\left(\varepsilon_{f, F \max }\right)$.

No âmbito de avaliar a eficácia do sistema de reforço com mantas de CFRP aplicadas segundo a técnica EBR, a Figura 15 apresenta uma comparação entre as curvas força versus deslocamento vertical das vigas ensaiadas nas idades de 42 e 87 dias, mantidas em ambiente laboratorial ou expostas ao protocolo de acondicionamento.

Pela análise das curvas tensão versus deformação das vigas sem reforço (V1/V2_LAB_0_14d V1/V2_LAB_0_1000h e V1/V2_ACP_0_1000h), as quais são apresentadas nas Figuras 15a-c-e, é possível perceber que, independentemente do ambiente de exposição, todas apresentaram os três estádios de comportamento típicos de elementos submetidos à flexão: o primeiro representa o concreto não fissurado, com comportamento elástico-linear; o segundo corresponde ao concreto fissurado com o aço no regime elástico, com redução da rigidez do elemento; e o terceiro equivale ao concreto fissurado com escoamento da armadura longitudinal de tração, apresentando patamar bem definido.

Pela análise das curvas tensão versus deformação das vigas reforçadas, (V1/V2_LAB_CFRP_14d V1/V2 LAB CFRP 1000h e V1/V2 ACP CFRP 1000h), apresentadas nas Figuras 15b-d-f, nota-se que, independentemente do ambiente de exposição, os elementos apresentam os mesmos três estádios de comportamento das vigas sem reforço. Porém, após o escoamento do aço, verifica-se que o sistema de reforço contribui para o aumento da capacidade de carga. Além disso, verifica-se redução do deslocamento vertical médio de 9,7\% para as vigas reforçadas devido à redução da fissuração que o sistema de reforço promove, restringindo a redução da inércia do elemento e influenciando na rigidez dos elementos. Por fim, o traçado linear da curva (sem patamar de escoamento) é consequência do comportamento elástico linear do compósito de CFRP.

A análise do comportamento dos elementos foi realizada considerando três cenários distintos:

(a) ambiente laboratorial e idade de 45 dias, considerado como referência para as demais análises;

(b) ambiente laboratorial e idade de 87 dias; e

(c) tanques de imersão seguindo o protocolo de acondicionamento acelerado e idade também igual a 87 dias.

Tabela 2 - Resumo dos resultados obtidos nos ensaios das vigas

\begin{tabular}{|c|c|c|c|c|c|c|c|c|c|c|c|c|c|c|}
\hline \multirow{2}{*}{ 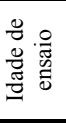 } & \multirow{2}{*}{ Vigas } & \multicolumn{4}{|c|}{$\begin{array}{l}\text { Escoamento da armadura } \\
\text { longitudinal }\left(\varepsilon_{s y}\right)\end{array}$} & \multicolumn{4}{|c|}{$\begin{array}{c}\text { Esmagamento do concreto } \\
\left(\varepsilon_{c, e s m}\right)\end{array}$} & \multicolumn{5}{|c|}{ Máxima força registrada } \\
\hline & & $\begin{array}{c}F_{s y} \\
(\mathbf{k N})\end{array}$ & $\begin{array}{c}u_{s y} \\
(\mathbf{m m})\end{array}$ & $\begin{array}{l}\varepsilon_{c, s y} \\
\mathbf{( \% )}\end{array}$ & $\begin{array}{l}\varepsilon_{f, s y} \\
(\% \mathbf{\%})\end{array}$ & $\begin{array}{l}F_{c, \text { esm }} \\
(\mathbf{k N})\end{array}$ & $\begin{array}{l}u_{e s m} \\
(\mathbf{m m})\end{array}$ & $\begin{array}{l}\varepsilon_{s, \text { esm }} \\
\text { (\%) }\end{array}$ & $\begin{array}{c}\varepsilon_{f, e s m} \\
(\% \mathbf{0})\end{array}$ & $\begin{array}{l}F_{\max } \\
(\mathbf{k N})\end{array}$ & $\begin{array}{c}u, F_{\max } \\
(\mathbf{m m})\end{array}$ & $\begin{array}{l}\varepsilon_{S, F \max } \\
\text { (\%) }\end{array}$ & $\begin{array}{c}\varepsilon_{c, F \max } \\
(\% \mathbf{\%})\end{array}$ & $\begin{array}{c}\varepsilon_{f, F \max } \\
(\% \mathbf{\%})\end{array}$ \\
\hline \multirow{4}{*}{ 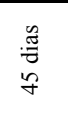 } & V1_LAB_0_14d & 22,9 & 10,7 & $-2,2$ & ---- & 23,6 & 15,3 & 5,9 & ---- & 25,3 & 36,2 & m.d. & m.d. & --- \\
\hline & V2_LAB_0_-14d & 24,6 & 13,2 & m.d. & ----- & ---- & ---- & ---- & ----- & 26,5 & 35,0 & m.d. & m.d. & ----- \\
\hline & V1_LAB_CFRP_14d & 33,7 & 10,5 & $-1,0$ & 3,7 & m.d. & m.d. & m.d. & m.d. & 41,6 & 21,4 & m.d. & m.d. & 9,4 \\
\hline & V2_LAB_CFRP_14d & 30,3 & 10,8 & 0,9 & 3,7 & m.d. & m.d. & m.d. & m.d. & 38,2 & 20,8 & m.d. & m.d. & 8,8 \\
\hline \multirow{4}{*}{ 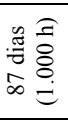 } & V1_LAB_0_1000h & 25,7 & 11,5 & $-1,2$ & ----- & 26,8 & 15,6 & m.d. & ----- & 28,4 & 35,0 & $-5,1$ & m.d. & ----- \\
\hline & V2_LAB_0_1000h & 26,0 & 11,3 & $-1,2$ & ----- & 27,5 & 17,9 & m.d. & ---- & 28,9 & 36,5 & $-4,8$ & m.d. & ----- \\
\hline & V1_LAB_CFRP_1000h & 30,0 & 12,2 & $-0,5$ & 4,6 & m.d. & ---- & ----- & ----- & 38,8 & 24,2 & m.d. & 14,8 & 11,1 \\
\hline & $\mathrm{V} 2{ }^{-} \mathrm{LAB}^{-} \mathrm{CFRP} 1000 \mathrm{~h}$ & 30,7 & 10,2 & $-1,5$ & 3,5 & 36,2 & 15,2 & 3,9 & 5,9 & 39,4 & 22,5 & $-4,0$ & m.d. & 8,3 \\
\hline \multirow{4}{*}{ 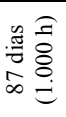 } & V1_ACP_0_1000h & 25,3 & 11,4 & $-0,8$ & ----- & \begin{tabular}{|l|}
----- \\
\end{tabular} & ----- & m.d. & ----- & 26,7 & 30,5 & m.d. & m.d. & ----- \\
\hline & V2_ACP_0_1000h & 25,5 & 11,2 & $-1,5$ & ----- & 26,3 & 22,6 & m.d. & ----- & 26,9 & 31,8 & m.d. & m.d. & ----- \\
\hline & V1_ACP_CFRP_1000h & 29,1 & 10,6 & m.d. & 2,8 & ---- & ----- & ----- & ----- & 36,5 & 23,9 & m.d. & m.d. & 7,5 \\
\hline & $\mathrm{V} 2{ }^{-} \mathrm{ACP}{ }^{-} \mathrm{CFRP} 1000 \mathrm{~h}$ & 28,9 & 10,9 & $-1,1$ & 2,9 & 33,0 & 15,9 & 2,5 & 5,7 & 34,5 & 20,6 & m.d. & m.d. & 6,5 \\
\hline
\end{tabular}

Fonte: Sarti Júnior (2020).

Nota: m.d. = extensômetros mecanicamente danificados 
Figura 15 - Força versus deslocamento das vigas de referência sem reforço e reforçadas

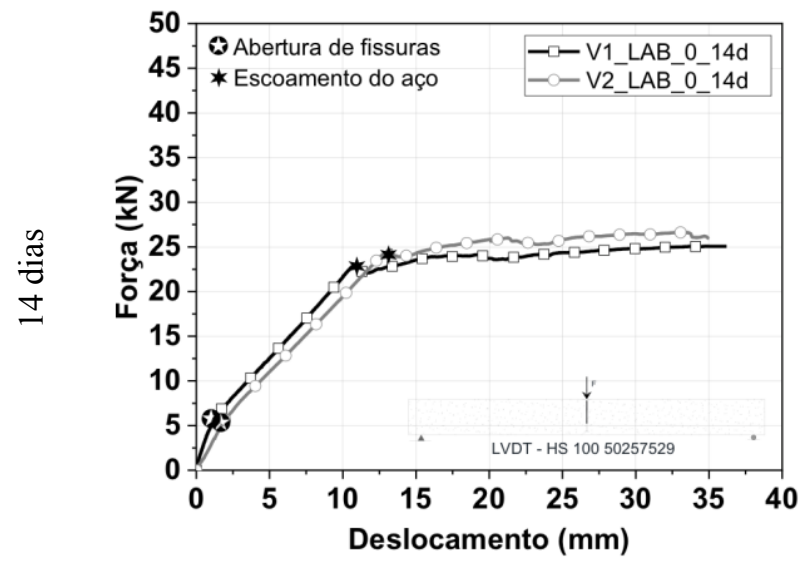

(a)

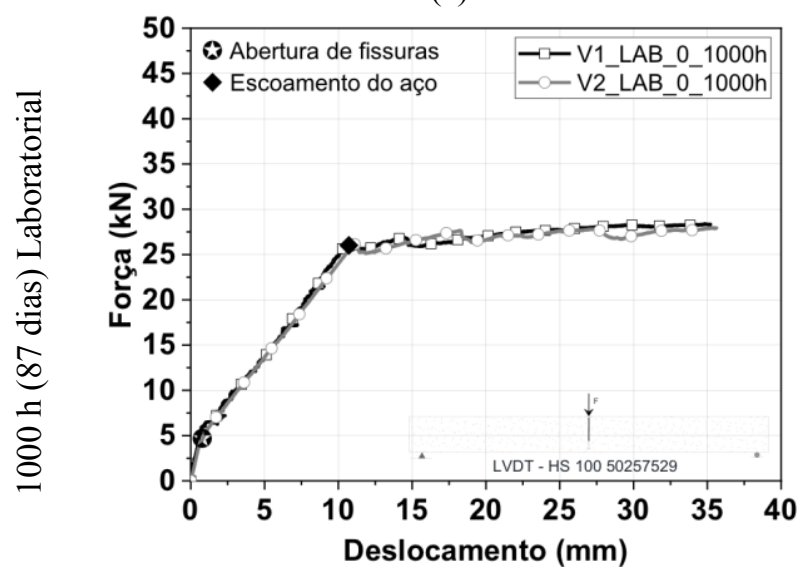

(c)

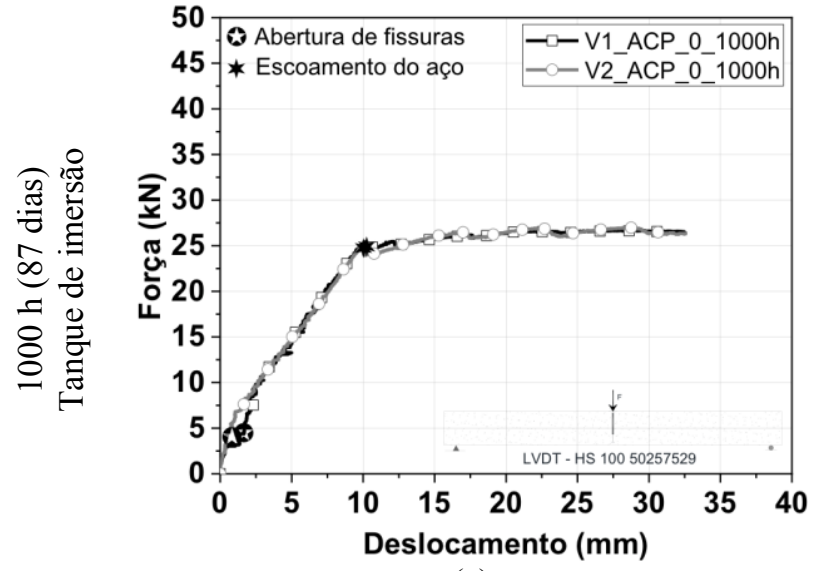

(e)

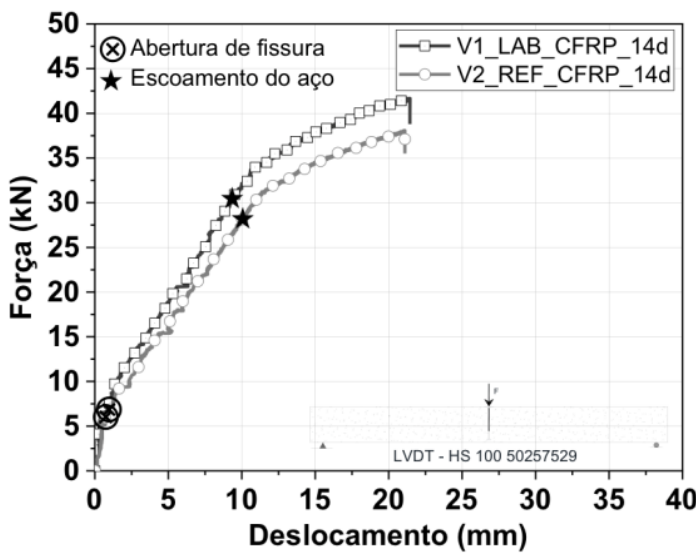

(b)

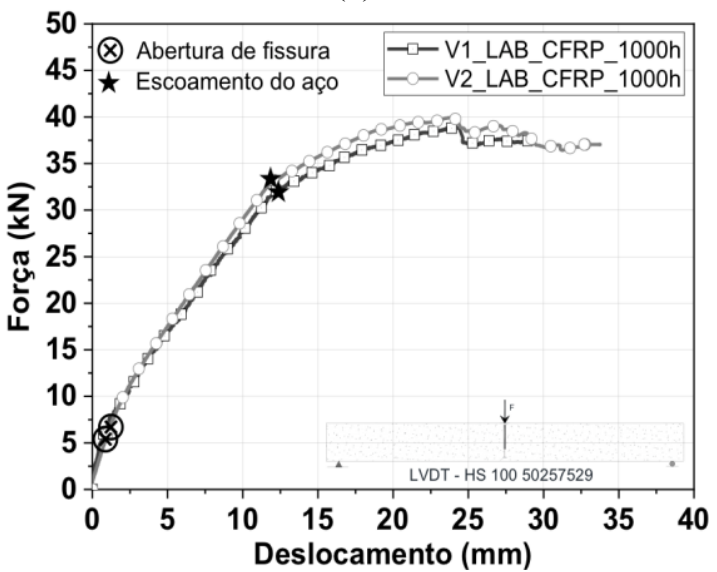

(d)

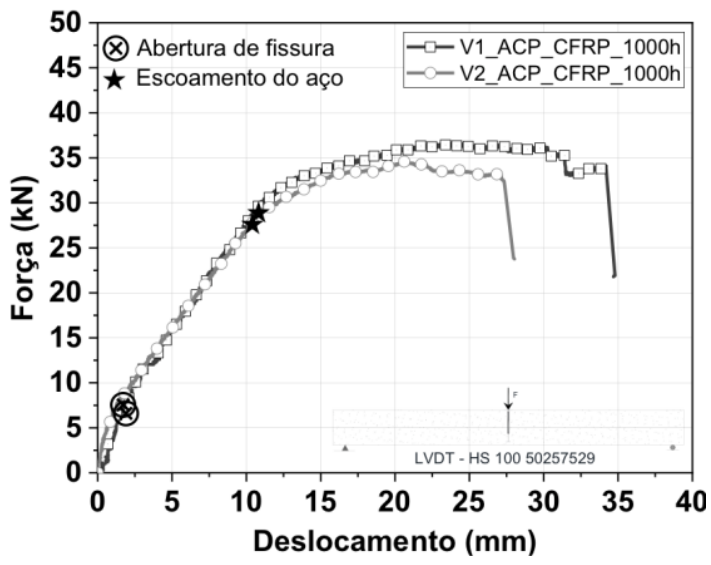

(f)

Fonte: Sarti Júnior (2020).

\section{Ambiente laboratorial - 45 dias - 14 dias após a aplicação do sistema de reforço}

Com relação à abertura de fissuras, as vigas sem reforço (Figura 15a) e reforçadas (Figura 15b) apresentam a primeira fissura nos primeiros pontos de inflexão para uma força média de $5,3 \mathrm{kN}$ e de $5,8 \mathrm{kN}$ e deslocamento médio de $1,4 \mathrm{~mm}$ e $0,5 \mathrm{~mm}$ respectivamente, com redução na rigidez do conjunto devido à fissuração do concreto a partir desses pontos. Em relação ao início do escoamento do aço, verifica-se que este ocorreu para forças e deslocamentos médios de $23,8 \mathrm{kN}$ e de $12 \mathrm{~mm}$ para as vigas sem reforço e de 32 $\mathrm{kN}$ e de $10,7 \mathrm{~mm}$ para as vigas reforçadas respectivamente. Verifica-se, para o início do escoamento, que o sistema de reforço proporcionou um aumento médio da capacidade de carga de $34,5 \%$ e que, devido ao 
aumento da rigidez proporcionado pela restrição da fissuração dos elementos reforçados, promoveu a redução do deslocamento vertical médio em $10,8 \%$. As vigas sem reforço e reforçadas apresentaram força média máxima registrada de $25,9 \mathrm{kN}$ e de $39,9 \mathrm{kN}$, indicando um aumento médio da capacidade de carga de $54 \%$ em relação às vigas não reforçadas. Para além disso, redução do deslocamento vertical médio em $40,7 \%$ foi obtida com o uso do sistema de reforço. A ruína, que antes era dúctil e governada pela deformação da armadura longitudinal, passou a ocorrer de maneira quase frágil com o descolamento da manta de CFRP aderida ao substrato de concreto.

\section{Ambiente laboratorial - 87 dias $(1.000$ h ou 42 dias)}

Com relação à abertura de fissuras, as vigas sem reforço (Figura 15c) e reforçadas (Figura 15d) apresentam a primeira fissura nos primeiros pontos de inflexão para uma força média de $7,2 \mathrm{kN}$ e de $6,9 \mathrm{kN}$ e deslocamento médio de $1,6 \mathrm{~mm}$ e de $1,6 \mathrm{~mm}$ respectivamente, com redução na rigidez do conjunto devido à fissuração do concreto a partir desses pontos.

Com relação ao início do escoamento do aço, verifica-se que este ocorreu para força e deslocamentos médios de $25,9 \mathrm{kN}$ e de $11,4 \mathrm{~mm}$ para as vigas sem reforço e de $30,4 \mathrm{kN}$ e de $11,2 \mathrm{~mm}$ para a vigas reforçadas respectivamente. Verifica-se, para o início do escoamento, que o sistema de reforço proporcionou um aumento médio da capacidade de carga de 17,4\%, inferior ao obtido para viga com idade de 45 dias. Com relação ao deslocamento vertical, valores similares foram obtidos. Com relação à força média máxima, as vigas sem reforço e reforçadas apresentaram valores de $28,6 \mathrm{kN}$ e de $39,1 \mathrm{kN}$, indicando um aumento médio da capacidade de carga de $36,7 \%$ em relação às vigas não reforçadas. Para além disso, redução do deslocamento vertical médio em $34,7 \%$ foi obtida com o uso do sistema de reforço. Tal como anteriormente apresentado, a ruptura deu-se de maneira quase frágil com o descolamento da manta de CFRP aderida ao substrato de concreto.

\section{Tanques de imersão - 87 dias (1.000 h ou 42 dias)}

Com relação à abertura de fissuras, as vigas sem reforço (Figura 15e) e reforçadas (Figura 15f) apresentam a primeira fissura nos primeiros pontos de inflexão das curvas mostradas na Figura 15, a uma força média de $7,4 \mathrm{kN}$ e de 7,6 $\mathrm{kN}$ e deslocamento médio de 1,4 e de $3,2 \mathrm{~mm}$ respectivamente, com redução na rigidez do conjunto devido à fissuração do concreto a partir desses pontos.

Com relação ao início do escoamento do aço, verifica-se que este ocorreu para força e deslocamentos médios de $25,4 \mathrm{kN}$ e de $14,3 \mathrm{~mm}$ para as vigas sem reforço e de $29 \mathrm{kN}$ e de $10,8 \mathrm{~mm}$ para a vigas reforçadas respectivamente. Verifica-se, para o início do escoamento, redução do deslocamento vertical médio em $24,5 \%$ com o uso do sistema de reforço. Para além disso, aumento médio da capacidade de carga de $14,2 \%$ foi obtido com o uso do reforço.

Com relação à força média máxima, as vigas sem reforço e reforçadas apresentaram valores de $26,8 \mathrm{kN}$ e de $35,5 \mathrm{kN}$, indicando um aumento médio da capacidade de carga de 32,5\% em relação às vigas não reforçadas, respectivamente. Para além disso, redução do deslocamento vertical médio em $28,6 \%$ foi obtida com o uso do sistema de reforço. Tal como anteriormente apresentado, a ruptura deu-se de maneira quase frágil com o descolamento da manta de CFRP aderida ao substrato de concreto.

\section{Comparação entre ambientes de exposição}

A Figura 16a apresenta as curvas médias força versus deslocamento para as vigas mantidas em ambiente laboratorial e expostas ao protocolo de acondicionamento para avaliação da durabilidade (Figura 16b), tal como apresentado na norma ACI 440.9R (AMERICAN..., 2015).

Com relação às vigas sem reforço e tendo a idade de 45 dias como referência (LAB_0_14d), percebe-se um aumento de $37 \%$ na força que leva à abertura das primeiras fissuras visíveis nos elementos, mas com deslocamentos equivalentes. Após a fissuração, é verificada a redução na rigidez do conjunto; entretanto, ela é mais evidenciada na viga com idade de 45 dias devido a sua menor resistência do concreto e, também, devido à abertura de fissuras precoce.

As vigas com idade de 87 dias apresentaram um pequeno aumento da capacidade portante no momento de início de escoamento (8,8\% e 6,7\% para LAB_0_1000h e ACP_0_1000h respectivamente). Para além disso, deslocamento similar foi observado para as vigas, independentemente da idade e do ambiente de exposição.

288 Dalfré, G. M.; Sarti Júnior, L. A.; Araújo, C. J. R. V. 
Figura 16 - Comparação entre diagramas força versus deslocamento das vigas de referência e reforçadas

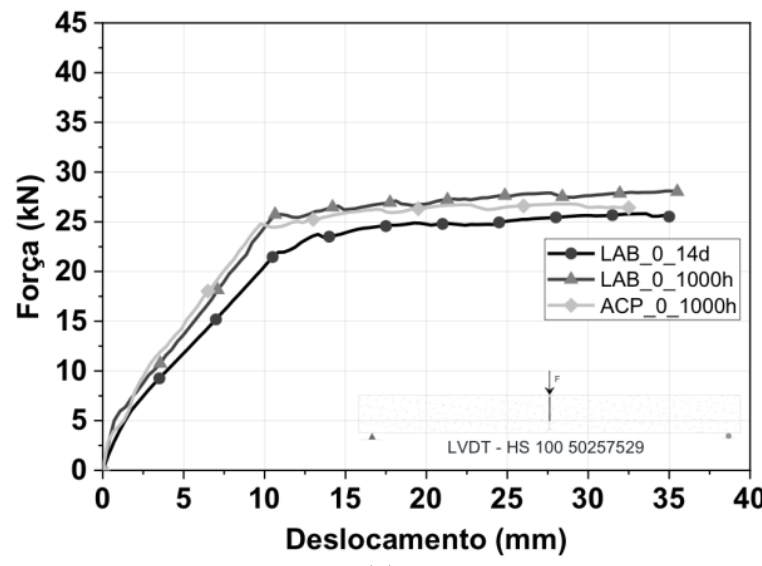

(a)

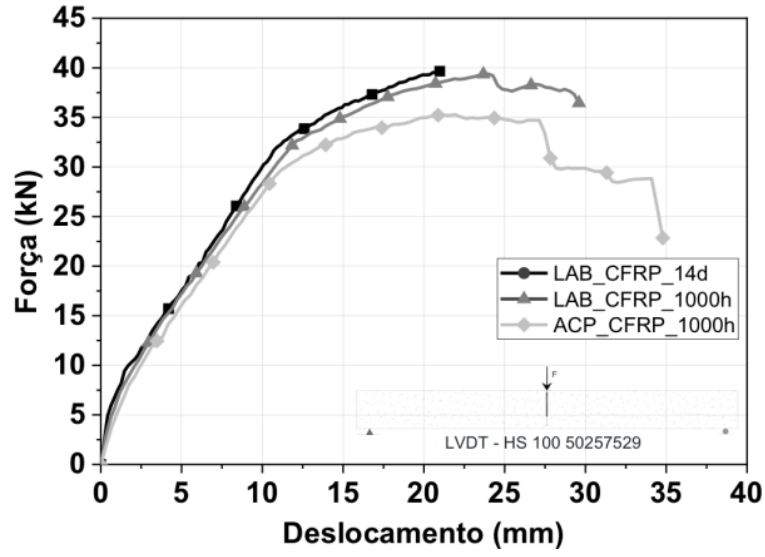

(b)

Fonte: Sarti Júnior (2020).

Considerando a força máxima atingida pelas vigas, estas apresentaram um aumento de carga de 7,8\% e deslocamentos similares em relação às vigas com idade de 45 dias. Com relação às vigas com idade de 87 dias, ambas apresentam comportamentos similares. Assim, verifica-se que para o ciclo de $1.000 \mathrm{~h}$ as vigas apresentaram comportamento similar independentemente do ambiente de exposição.

Com relação às vigas reforçadas e também tendo a idade de 45 dias como referência, percebe-se aumento de $19 \%$ e de $31 \%$ nas forças que levam ao surgimento das primeiras fissuras, acompanhado do aumento do deslocamento vertical em $220 \%$ e $540 \%$ para as vigas LAB_CFRP_1000h e ACP_CFRP_1000h respectivamente.

Com relação ao início do escoamento, todas as vigas ensaiadas com idade de 87 dias apresentaram capacidade portante similar. Entretanto, as vigas reforçadas e expostas à degradação acelerada apresentaram deslocamento $4 \%$ inferior às vigas mantidas em ambiente laboratorial.

Com relação à força máxima, e considerando a viga LAB_CFRP_14d como referência, percebe-se que, embora a viga LAB_CFRP_1000h apresente mesma capacidade resistente, sua ductilidade é $11 \%$ superior.

Considerando-se as vigas reforçadas com idade de 87 dias e tendo em vista o comportamento obtido para a viga mantida em ambiente laboratorial (LAB_CFRP_1000h), percebe-se que o protocolo apresentado pela norma ACI 440.9R (AMERICAN..., 2015) levou à dēgradação do sistema de reforço. Nesse sentido, a viga LAB_ACP_1000h apresentou redução de $11 \%$ da força média máxima.

Com relação às deformações registradas no compósito de CFRP e tomando como referência as vigas LAB_CFRP_14d e LAB_CFRP_1000h, observa-se que o sistema de reforço aplicado nas vigas expostas à degradação acelerada apresentou valores inferiores tanto no início do escoamento como na máxima força resistida pelos elementos. Tal comportamento é similar aos observados por Pan, Xian e Silva (2015), que verificaram que a umidade constante e temperatura de $50{ }^{\circ} \mathrm{C}$ promoveram alterações na ligação entre concreto, adesivo e FRP, provocando o descolamento prematuro do sistema de reforço. Para além disso, Kabir, Shrestha e Samali (2016), Zheng et al. (2016) e Silva et al. (2016) observaram em seus estudos que a presença de umidade e temperatura são os principais fatores que provocam o enfraquecimento da ligação compósito-concreto, principalmente quando expostos a longos períodos.

Com relação à ruptura, todas as vigas apresentaram descolamento da manta de CFRP aderida ao substrato de concreto. Entretanto, as vigas com idade de 87 dias apresentam aumento do deslocamento vertical médio na ruptura de 45,5\%. Porém, a viga ACP_CFRP_1000h apresenta maior variabilidade de deslocamentos registrados na ruptura, provavelmente devido à degradação das resinas epoxídicas utilizadas na adesão do sistema de reforço ao substrato, tal como verificado na Figura 16. 
Figura 17 - Curvas médias para força versus deslocamento para as vigas expostas ao intemperismo, sem proteção, por um período de 6 meses e expostas ao protocolo de degradação acelerada do presente trabalho

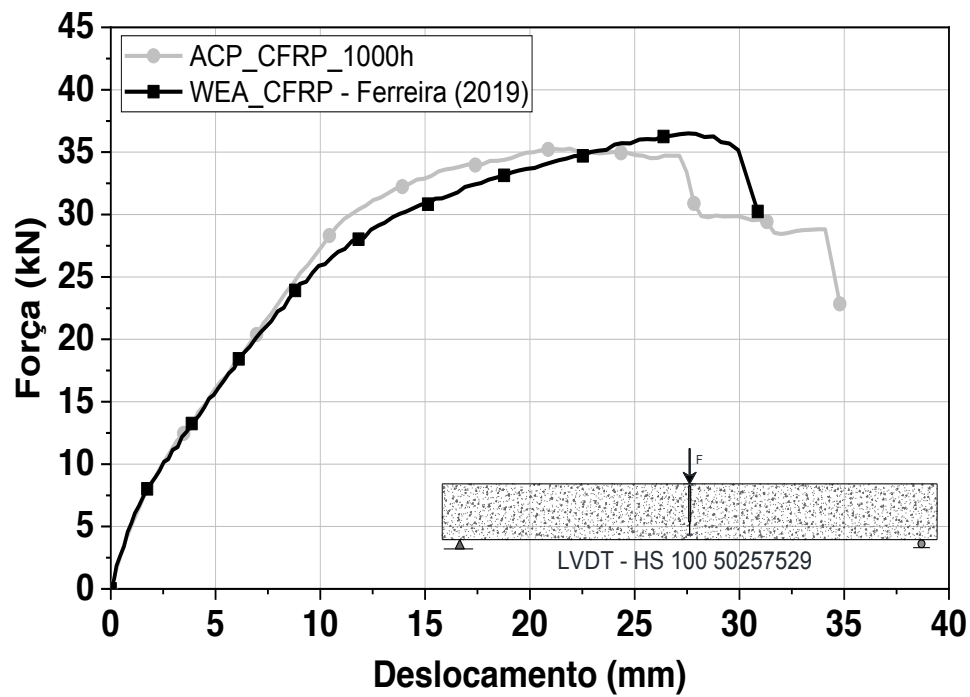

Fonte: Sarti Júnior (2020).

\section{Comparação do protocolo de degradação acelerado com ensaios de intemperismo natural}

A Figura 17 apresenta as curvas médias força versus deslocamento para as vigas expostas ao intemperismo, sem proteção, por um período de 6 meses e expostas ao protocolo de acondicionamento baseado na norma ACI 440.9R (AMERICAN..., 2015). Os resultados apresentados por Ferreira (2019) e os obtidos neste trabalho indicam redução da força máxima de aproximadamente $10 \%$ e $11 \%$ após a exposição ao intemperismo ou com a aplicação do protocolo acelerado respectivamente. Nesse sentido, uma análise simplista baseada na força máxima registrada nos elementos analisados indica que um ciclo de $1.000 \mathrm{~h}$ do protocolo de acondicionamento corresponde a um período de degradação natural ante o intemperismo, sem proteção do sistema de reforço, de aproximadamente 6 meses.

Com relação ao deslocamento vertical, uma grande dispersão foi verificada no momento da ruptura tanto para os elementos expostos ao intemperismo quanto para os mantidos em degradação acelerada.

Informa-se que a pesquisa aqui apresentada será complementada com os ensaios de degradação acelerada a serem realizados para os ciclos de $3.000 \mathrm{~h}$ (125 dias) e $10.000 \mathrm{~h}$ (417 dias), enquanto as vigas expostas ao intemperismo serão ensaiadas para a idade de 2 anos. Assim, uma melhor correlação será futuramente obtida, levando a um melhor entendimento do comportamento em longo prazo de elementos reforçados e ajudando na obtenção de um modelo de previsão de vida útil para sistemas de reforço EBR-CFRP aplicados em vigas solicitadas a flexão.

\section{Conclusões}

Este trabalho apresenta um programa experimental que visa avaliar o comportamento no longo prazo de sistemas de reforço EBR-CFRP. Para isso, dois ambientes de exposição foram adotados nesta pesquisa: laboratorial, o qual serviu de referência para as demais análises; e tanque de imersão do sistema de reforço baseado no protocolo de acondicionamento, recomendado pela norma ACI 440.9R (AMERICAN..., 2015), visando a possível degradação dos FRP. Os resultados obtidos permitiram a obtenção das conclusões a seguir:

(a) com relação aos materiais utilizados no sistema de reforço, a resina A (primer) mantida em laboratório apresentou módulos de elasticidade e tensão máximas similares para as idades de 14 dias e 42 dias.

Entretanto, as resinas acondicionadas nos tanques e ensaiadas aos 42 dias apresentaram reduções de $63 \%$ da força máxima e de $61 \%$ do módulo de elasticidade. Com relação à resina B (saturação), tendo como referência a idade de 14 dias, reduções de $22,6 \%$ e de $47,6 \%$ na tensão máxima e no módulo de elasticidade foram observadas para a resina mantida em ambiente laboratorial e ensaiada com idade de 42 dias. Quando

290 Dalfré, G. M.; Sarti Júnior, L. A.; Araújo, C. J. R. V. 
exposta ao protocolo de acondicionamento, e tendo como referência o ambiente laboratorial e idade de 42 dias, redução de $50 \%$ da tensão máxima foi obtida, sem alteração do módulo de elasticidade;

(b) com relação aos compósitos, verifica-se que os corpos de prova apresentaram um comportamento elástico linear até sua ruptura, típico de materiais frágeis. Com relação aos resultados obtidos, reduções de $6 \%$ da tensão máxima e do módulo de elasticidade foram verificados na comparação entre os ensaios segundo o protocolo de acondicionamento e o ambiente laboratorial;

(c) a eficiência da técnica EBR no aumento da capacidade de carga de vigas de concreto armado reforçadas à flexão com mantas de CFRP foi verificada por meio do aumento significativo na capacidade de carga e no incremento da rigidez dos elementos reforçados;

(d) todas as vigas sem reforço (LAB/ACP_0_1000h) ensaiadas na idade de 87 dias apresentaram comportamento força versus deslocamento similar, independentemente do meio de exposição. Assim, percebe-se que não houve degradação dos elementos não reforçados ante o protocolo de acondicionamento utilizado;

(e) para as vigas reforçadas e ensaiadas com idade de 87 dias, e considerando-se o comportamento obtido para a viga mantida em ambiente laboratorial (LAB_CFRP_1000h), percebe-se que o protocolo apresentado pela ACI 440.9R (AMERICAN..., 2015) levou à degradação do sistema de reforço. Nesse sentido, as vigas LAB_ACP_1000h apresentaram redução de 11\% da força média máxima;

(f) com relação à ruptura, todas as vigas apresentaram descolamento da manta de CFRP aderida ao substrato de concreto. As vigas com idade de 87 dias apresentam aumento do deslocamento vertical médio na ruptura de $45,5 \%$, enquanto as vigas expostas ao protocolo de acondicionamento apresentam maior variabilidade de deslocamentos registrados na ruptura, provavelmente devido à degradação das resinas epoxídicas utilizadas na adesão do sistema de reforço ao substrato; e

(g) tendo em vista a correlação com os resultados obtidos em outro programa experimental em andamento, uma análise simplista, baseada somente na força máxima registrada, indica que um ciclo de $1.000 \mathrm{~h}$ do protocolo de acondicionamento recomendado pela norma ACI 440.9R (AMERICAN..., 2015) corresponde a um período de degradação natural do sistema de reforço devido ao intemperismo de aproximadamente 6 meses.

\section{Referências}

AMERICAN CONCRETE INSTITUTE. 440.2R: guide for the design and construction of externally bonded FRP systems for strengthening concrete structures. Farmington Hills, 2017.

AMERICAN CONCRETE INSTITUTE. 440.9R: guide to accelerated conditioning protocols for durability assessment of internal and external fiber-reinforced polymer (FRP) reinforcement. Farmington Hills, 2015.

ASSOCIAÇÃO BRASILEIRA DE NORMAS TÉCNICAS. NBR 5738: concreto: procedimento para moldagem e cura de corpos de prova. Rio de Janeiro, 2015.

ASSOCIAÇÃO BRASILEIRA DE NORMAS TÉCNICAS. NBR 5739: concreto: ensaio de compressão de corpos de prova cilíndricos. Rio de Janeiro, 2018 b.

ASSOCIAÇÃO BRASILEIRA DE NORMAS TÉCNICAS. NBR 6118: projeto de estruturas de concreto: procedimento. Rio de Janeiro, 2014.

ASSOCIAÇÃO BRASILEIRA DE NORMAS TÉCNICAS. NBR 6892-1: materiais metálicos: ensaio de tração: parte 1: método de ensaio à temperatura ambiente. Rio de Janeiro, 2018a.

ASSOCIAÇÃO BRASILEIRA DE NORMAS TÉCNICAS. NBR 8522: concreto: determinação dos módulos elásticos de elasticidade e de deformação à compressão. Rio de Janeiro, 2018c.

CROMWELL, J. R.; HARRIES, K. A.; SHAHROOZ, B. M. Environmental durability of Externally bonded FRP materials intended for repair of concrete structures. Construction and Building Materials, v. 25, n. 5, p. 2528-2539, 2010 .

DALFRÉ, G. et al. Avaliação do comportamento de resinas epoxídicas submetidas a ciclos de umidade. In: SIMPÓSIO PARANAENSE DE PATOLOGIA DAS CONSTRUÇÕES, 4., Curitiba, 2019. Anais [...] Curitiba, 2019. 
DALFRÉ, G. M. Estudo da degradação da aderência de compósitos colados a superfícies de concreto armado quando submetidos a ciclos de umidade e carbonatação. Relatório Técnico - Projeto Universal CNPq 483720/2013-5, CNPq, 2016.

DALFRÉ, G. M. Flexural and shear strengthening of RC elements. Guimarães, 2013. Tese (Doutorado em Engenharia Civil) - Universidade do Minho, Guimarães, 2013.

ESCOBAL, A. D. Caracterização da degradação de adesivos estruturais utilizados em sistemas de reforço de estrutura de concreto armado. Relatório científico de progresso. Projeto FAPESP - Processo 2016/20025-6. São Carlos, SP, 2017.

FÉDÉRATION INTERNACIONALE DU BETÓN. Bulletin 90: externally applied FRP reinforcement for concrete structures. Lausanne, 2019.

FERNANDES, P. M. G. Bond behavior of NSM CFRP-concrete systems: durability and quality control. Guimarães, 2016.Tese (Doutorado em Engenharia Civil) - Universidade do Minho, Guimarães, 2016.

FERREIRA, D. C. Avaliação da degradação de vigas reforçadas com FRP quando expostas ao intemperismo. São Carlos, 2019. Dissertação (Mestrado em Engenharia Civil) - Universidade Federal de São Carlos, São Carlos, 2019.

FRIGIONE, M. Fiber reinforced polymers in civil engineering: durability issues. Advanced Materials Research, v. 1129, p. 283-289, 2015.

HOMAM, S. M.; SHEIKH, S. A. Durability of fiber reinforced polymers used in concrete structures. In: INTERNATIONAL CONFERENCE ON ADVANCED MATERIALS IN BRIDGES AND STRUCTURES, 3., Ottawa, 2000. Proceedings [...] Ottawa, Canada, 2000. p. 751-758.

INTELLIGENT SENSING FOR INNOVATIVE STRUCTURES. Durability of FRP composites for construction. Educational module $n^{\circ} 8$ (student notes). Manitoba: The Canadian Network of Centres of Excellence on Intelligent Sensing for Innovative Structures, 2006.

INTERNATIONAL ORGANIZATION FOR STANDARDIZATION. ISO 527-1: plastics: determination of tensile properties: part 1: general principles. Geneva, 2012a.

INTERNATIONAL ORGANIZATION FOR STANDARDIZATION. ISO 527-2: plastics: determination of tensile properties: part 2: test conditions for molding and extrusion plastics. Geneva, 2012b.

INTERNATIONAL ORGANIZATION FOR STANDARDIZATION. ISO 527-5: plastics: determination of tensile properties: part 5: test conditions for unidirectional fibre-reinforced plastic composites. Geneva, 2009.

KABIR, M. I.; SHRESTHA, R.; SAMALI, B. Fracture properties of CFRP-concrete bond subjected to three environmental conditions. Journal of Composites for Construction, v. 20, n. 1, aug. 2016.

KARBHARI, V. M. Durability of composites for civil structural applications. Cambridge: Woodhead, 2007.

NORDIN, H. Flexural strengthening of concrete structures with prestressed near surface mounted CFRP rods. Luleå, 2003. 143 f. Tese (Doutorado em Engenharia Civil) - Universidade da Tecnologia Lulea, Luleå, 2003.

OKBA, S. H. et al. Effect of thermal exposure on the mechanical properties of polymer adhesives.

Construction and Building Materials, v. 135, p. 490-504, 2017.

OLIVEIRA, M. C. Análise do comportamento estrutural e durabilidade de pilares de concreto armado reforçados com mantas de GFRP. São Carlos, 2019. Dissertação (Mestrado em Engenharia Civil) Universidade Federal de São Carlos, São Carlos, 2019.

PAN, Y.; XIAN, G.; SILVA, M. A. G. Effects of water immersion on the bond behavior between CFRP plates and concrete substrate. Construction and Building Materials, v. 101, p. 326-337, 2015.

REZAZADEH, M.; BARROS, J. A. O.; RAMEZANSEFAT, H. End concrete cover separation in RC structure strengthened in flexure with NSM FRP: Analytical Design Approach. Engineering Structures, v. 128, p. 415-427, 2016.

SARTI JÚNIOR, L. A. Durabilidade de vigas de concreto armado reforçadas com mantas de CFRP submetidas a degradação acelerada. São Carlos, 2020. Dissertação (Mestrado em Engenharia Civil) Universidade Federal de São Carlos, São Carlos, 2020. 
SILVA, P. et al. Effects of different environmental conditions on the mechanical characteristics of a structural epoxy. Composites Part B, v. 88, p. 55-63, 2016.

YANG, Q.; XIAN, G.; KARBHARI, V. M. Hygrothermal ageing of an epoxy adhesive used in FRP strengthening of concrete. Journal of Applied Polymer Science, v. 107, n. 4, p. 2607-2617, 2008.

$\mathrm{ZHAO}$, J. et al. Deterioration of basic properties of the materials in FRP-strengthening RC structures under ultraviolet exposure. Polymers, v. 9, 2017.

ZHENG, X. H. et al. Experimental study on bond behavior of FRP-concrete interface in hygrothermal environment. International Journal of Polymer Science, v. 2016, article ID 5832130, 2016.

\section{Agradecimentos}

Os autores agradecem à empresa Metrodular, de Piracicaba, SP, pelo fornecimento das formas poliméricas utilizadas na concretagem das vigas, e ao Laboratório de Polímeros do Departamento de Engenharia de Materiais (DEMa) e ao Laboratório de Sistemas Estruturais (LSE) da Universidade Federal de São Carlos (UFSCar), pela realização dos ensaios desta pesquisa. O presente trabalho foi realizado com apoio do Instituto de Pesquisas Tecnológicas do Estado de São Paulo (IPT) e da Coordenação de Aperfeiçoamento de Pessoal de Nível Superior (Capes).

Gláucia Maria Dalfré

Programa de Pós-Graduação em Engenharia Civil | Universidade Federal de São Carlos | Rod. Washington Luiz, km 235 - SP 310 | São Carlos - SP - Brasil | CEP 13565-905 | Tel.: (16) 3351-9673 | E-mail: glaucia.dalfre@ufscar.br

Luiz Antônio Sarti Júnior

Programa de Pós-Graduação em Engenharia Civil | Universidade Federal de São Carlos | E-mail: luiz.sarti@gmail.com

Ciro José Ribeiro Villela Araújo

Departamento de Cidades, Infraestrutura e Meio Ambiente, Seção de Obras Civis I Instituto de Pesquisas Tecnológicas do Estado de São Paulo | Av. Prof. Almeida Prado, 532, Prédio 54 | São Paulo - SP - Brasil | CEP 05508-901 | Tel.: (11) 3767-4166 | E-mail: ciroaraujo@ipt.br

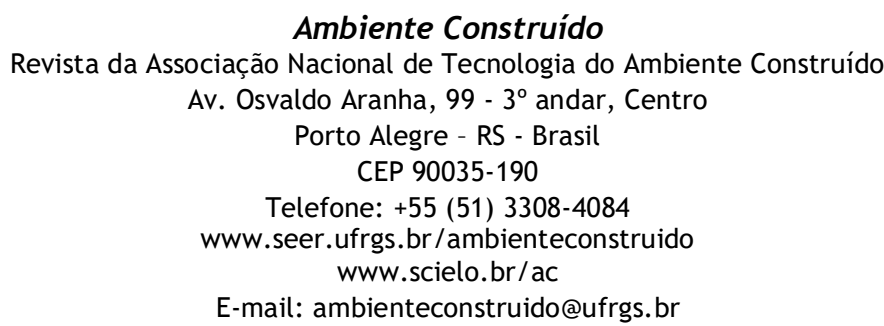

